\title{
Existence of local solutions for free boundary problems for viscous compressible barotropic fluids
}

\author{
by W. M. ZAJA̧CZKOWski (Warszawa)
}

\begin{abstract}
We prove the local existence of solutions for equations of motion of a viscous compressible barotropic fluid in a domain bounded by a free surface. The solutions are shown to exist in exactly those function spaces where global solutions were found in our previous papers $[14,15]$.
\end{abstract}

1. Introduction. We consider the motion of a viscous compressible barotropic fluid in a domain $\Omega_{t} \subset \mathbb{R}^{3}$ bounded by a free surface $S_{t}=\partial \Omega_{t}$. Let $v=v(x, t)$ be the velocity of the fluid, $\varrho=\varrho(x, t)$ the density, $f=f(x, t)$ the external force field per unit mass, $p=p(\varrho)$ the pressure, $\mu$ and $\nu$ the viscosity coefficients, $\sigma$ the surface tension coefficient and $p_{0}$ the external (constant) pressure. Then the problem is described by the following system (see [4], Chs. 1, 2, 5):

$$
\begin{array}{ll}
\varrho\left(v_{t}+v \cdot \nabla v\right)+\nabla p(\varrho)-\mu \Delta v-\nu \nabla \operatorname{div} v=\varrho f & \text { in } \widetilde{\Omega}^{T}, \\
\varrho t_{t}+\operatorname{div}(\varrho v)=0 & \text { in } \widetilde{\Omega}^{T}, \\
\left.\varrho\right|_{t=0}=\varrho_{0},\left.\quad v\right|_{t=0}=v_{0} & \text { in } \Omega, \\
\mathbb{T} \bar{n}-\sigma H \bar{n}=-p_{0} \bar{n} & \text { on } \widetilde{S}^{T}, \\
v \cdot \bar{n}=-\phi_{t} /|\nabla \phi| & \text { on } \widetilde{S}^{T},
\end{array}
$$

where $\phi(x, t)=0$ describes $S_{t}, \widetilde{\Omega}^{T}=\bigcup_{t \in(0, T)} \Omega_{t} \times\{t\}, \Omega_{t}$ is the domain of the drop at time $t \in(0, T), \Omega=\Omega_{0}$ is its initial domain, $\widetilde{S}^{T}=$ $\bigcup_{t \in(0, T)} S_{t} \times\{t\}, \bar{n}$ is the unit outward vector normal to the boundary $(\bar{n}=\nabla \phi /|\nabla \phi|)$, and $\mu, \nu, \sigma$ are constant coefficients. Moreover, thermodynamic considerations imply $\nu \geq 1 /(3 \mu)>0, \sigma>0$. The last condition $(1.1)_{5}$ means that the free boundary $S_{t}$ is built up of moving fluid particles.

1991 Mathematics Subject Classification: 35G30, 76N10, 35R35.

Key words and phrases: local existence, free boundary, anisotropic Sobolev spaces, viscous compressible barotropic fluids. 
Finally, $\mathbb{T}=\mathbb{T}(v, p)$ denotes the stress tensor of the form

(1.2) $\mathbb{T}=\left\{T_{i j}(v, p)\right\}=\left\{-p \delta_{i j}+\mu\left(\partial_{x_{i}} v_{j}+\partial_{x_{j}} v_{i}\right)+(\nu-\mu) \delta_{i j} \operatorname{div} v\right\}$

$$
\equiv\left\{-p \delta_{i j}\right\}+\left\{D_{i j}(v)\right\}
$$

where $i, j=1,2,3, \mathbb{D}=\mathbb{D}(v)=\left\{D_{i j}(v)\right\}$ is the deformation tensor and $H$ is the double mean curvature of $S_{t}$, which is negative for convex domains and can be expressed in the form

$$
H \bar{n}=\Delta_{S_{t}}(t) x, \quad x=\left(x_{1}, x_{2}, x_{3}\right),
$$

where $\Delta_{S_{t}}(t)$ is the Laplace-Beltrami operator on $S_{t}$. Let $S_{t}$ be determined by $x=x\left(s_{1}, s_{2}, t\right),\left(s_{1}, s_{2}\right) \in U \subset \mathbb{R}^{2}$, where $U$ is an open set. Then

$$
\Delta_{S_{t}}(t)=\frac{1}{\sqrt{g}} \partial_{s_{\alpha}} \frac{1}{\sqrt{g}} \widehat{g}_{\alpha \beta} \partial_{s_{\beta}}=\frac{1}{\sqrt{g}} \partial_{s_{\alpha}} \sqrt{g} g^{\alpha \beta} \partial_{s_{\beta}}, \quad \alpha, \beta=1,2,
$$

where the summation convention over repeated indices is assumed, $g=$ $\operatorname{det}\left\{g_{\alpha \beta}\right\}_{\alpha, \beta=1,2}, g_{\alpha \beta}=x_{\alpha} \cdot x_{\beta}$, where $x_{\alpha}=\partial_{s_{\alpha}} x$ and the dot denotes the scalar product in the Euclidean space, $\left\{g^{\alpha \beta}\right\}$ is the inverse matrix to $\left\{g_{\alpha \beta}\right\}$ and $\left\{\widehat{g}_{\alpha \beta}\right\}$ is the matrix of algebraic complements for $\left\{g_{\alpha \beta}\right\}$.

Let the domain $\Omega$ be given. Then by $(1.1)_{5}, \Omega_{t}=\left\{x \in \mathbb{R}^{3}: x=x(\xi, t)\right.$, $\xi \in \Omega\}$, where $x=x(\xi, t)$ is the solution of the Cauchy problem

$$
\frac{d x}{d t}=v(x, t),\left.\quad x\right|_{t=0}=\xi \in \Omega, \xi=\left(\xi_{1}, \xi_{2}, \xi_{3}\right) .
$$

Therefore the transformation $x=x(\xi, t)$ connects the Eulerian $x$ and the Lagrangian $\xi$ coordinates of the same fluid particle. Hence

$$
x=\xi+\int_{0}^{t} u(\xi, s) d s \equiv x(\xi, t),
$$

where $u(\xi, t)=v(x(\xi, t), t)$. Moreover, the kinematic boundary condition $(1.1)_{5}$ implies that the boundary $S_{t}$ is a material surface, so if $\xi \in S=S_{0}$, then $x(\xi, t) \in S_{t}$ and

$$
S_{t}=\{x: x=x(\xi, t), \xi \in S\} .
$$

In view of the continuity equation $(1.1)_{2}$ and $(1.1)_{5}$ the total mass $M$ is conserved and

$$
\int_{\Omega_{t}} \varrho(x, t) d x=M, \quad t \in[0, T],
$$

which is also a relation between $\varrho$ and $\Omega_{t}$.

We consider simultaneously two cases: $\sigma>0$ and $\sigma=0$. The aim of this paper is to prove local existence of solutions to problem (1.1). To prove the existence we use the Lagrangian coordinates. Therefore, we write problem 
(1.1) in the form

$$
\begin{array}{ll}
\eta u_{t}-\mu \nabla_{u}^{2} u-\nu \nabla_{u} \nabla_{u} \cdot u+\nabla_{u} q=\eta g & \text { in } \Omega^{T}=\Omega \times(0, T), \\
\eta_{t}+\eta \nabla_{u} \cdot u=0 & \text { in } \Omega^{T}, \\
\mathbb{T}_{u}(u, q) \bar{n}-\sigma \Delta_{S_{t}}(t) x(\xi, t)=-p_{0} \bar{n} & \text { on } S^{T}=S \times(0, T), \\
\left.u\right|_{t=0}=v_{0}(\xi) & \text { in } \Omega, \\
\left.\eta\right|_{t=0}=\varrho(\xi) & \text { in } \Omega,
\end{array}
$$

where $\eta(\xi, t)=\varrho(x(\xi, t), t), q(\xi, t)=p(x(\xi, t), t), g(\xi, t)=f(x(\xi, t), t)$, $\nabla_{u}=\partial_{x} \xi_{i} \nabla_{\xi_{i}}, \partial_{\xi_{i}}=\nabla_{\xi_{i}}, \mathbb{T}_{u}(u, q)=-q \delta+\mathbb{D}_{u}(u), \delta=\left\{\delta_{i j}\right\}_{i, j=1,2,3}$ is the unit matrix and $\mathbb{D}(u)=\left\{\mu\left(\partial_{x_{i}} \xi_{k} \nabla_{\xi_{k}} u_{j}+\partial_{x_{j}} \xi_{k} \nabla_{\xi_{k}} u_{i}\right)+(\nu-\mu) \delta_{i j} \nabla_{u} \cdot u\right\}$, with $\nabla_{u} \cdot u=\partial_{x_{i}} \xi_{j} \nabla_{\xi_{j}} u_{i}$, with summation over repeated indices.

Let $A$ be the Jacobi matrix of the transformation $x=x(\xi, t)$ with elements $a_{i j}=\delta_{i j}+\int_{0}^{t} \partial_{\xi_{j}} u_{i}(\xi, \tau) d \tau$. Let $0<M_{0}=$ const be given. Assuming $\left|\nabla_{\xi} u\right|_{\infty, \Omega^{T}} \leq M_{0}$ we obtain

$$
0<c_{1}\left(1-M_{0} t\right)^{3} \leq \operatorname{det}\left\{\partial_{\xi_{i}} x_{j}\right\} \leq c_{2}\left(1+M_{0} t\right)^{3}, \quad t \leq T,
$$

where $c_{1}, c_{2}$ are constants and $T$ is sufficiently small. Moreover, $\operatorname{det} A=$ $\exp \left(\int_{0}^{t} \nabla_{u} \cdot u d \tau\right)=\varrho / \eta$.

Since $S_{t}$ is determined (at least locally) by the equation $\phi(x, t)=0, S$ is described by $\left.\phi(x(\xi, t), t)\right|_{t=0} \equiv \widetilde{\phi}(\xi)=0$. Moreover, we have

$$
\bar{n}=\bar{n}(x(\xi, t), t)=\left.\frac{\nabla_{x} \phi(x, t)}{\left|\nabla_{x} \phi(x, t)\right|}\right|_{x=x(\xi, t)}, \quad \bar{n}_{0}=\bar{n}_{0}(\xi)=\frac{\nabla_{\xi} \widetilde{\phi}(\xi)}{\left|\nabla_{\xi} \widetilde{\phi}(\xi)\right|}
$$

The proof of existence of solutions of problem (1.8) is divided into the following steps. First we prove existence of solutions to the problem (see Section 4)

$$
\begin{array}{ll}
u_{t}-\mu \Delta_{\xi} u-\nu \nabla_{\xi} \nabla_{\xi} \cdot u=f_{1} & \text { in } \Omega^{T} \\
\Pi_{0} \mathbb{D}_{\xi}(u) \bar{n}_{0}=g_{1} & \text { on } S^{T} \\
\bar{n}_{0} \mathbb{D}_{\xi}(u) \bar{n}_{0}-\sigma \bar{n}_{0} \Delta_{S}(0) \int_{0}^{t} u(\tau) d \tau=g_{2}+\sigma \int_{0}^{t} h_{1}(\tau) d \tau & \text { on } S^{T}, \\
\left.u\right|_{t=0}=u_{0} & \text { in } \Omega
\end{array}
$$

where $\Pi_{0}$ is the projection defined by $\Pi_{0} g=g-\left(g \cdot \bar{n}_{0}\right) \bar{n}_{0}$ and $\mathbb{D}_{\xi}(u)=$ $\left\{\mu\left(\partial_{\xi_{i}} u_{j}+\partial_{\xi_{j}} u_{i}\right)+(\nu-\mu) \delta_{i j} \partial_{\xi_{k}} u_{k}\right\}$.

Next we prove existence of solutions to the problem (see Section 5) 


$$
\begin{array}{ll}
\eta u_{t}-\mu \nabla_{\omega}^{2} u-\nu \nabla_{\omega} \nabla_{\omega} \cdot u=F & \text { in } \Omega^{T}, \\
\mathbb{T}_{\omega}(u, q) \bar{n}-\sigma \Delta_{S_{t}}(t)\left(\xi+\int_{0}^{t} \omega(\xi, \tau) d \tau\right) & \\
=G+\sigma \int_{0}^{t} H(\tau) d \tau & \text { on } S^{T}, \\
\left.u\right|_{t=0}=v_{0} & \text { in } \Omega,
\end{array}
$$

where $\eta$ and $\omega$ are given functions.

Finally, by the method of successive approximations we show existence of solutions of problem (1.8) (see Section 6).

In Section 2 we introduce the necessary notation and present some auxiliary results.

In this paper we prove existence of solutions to problem (1.1) exactly in those classes in which global existence for this problem is shown (see [14, 15]). In [13] the local existence of solutions to (1.1) is proved in totally different anisotropic Sobolev spaces. Therefore the proofs from this paper and [13] are different in many details although the general idea is the same.

In this paper we tried to present numerous details of the proof because the result is fundamental for the considerations in $[9,10,11,14,15]$, where the local existence has already been assumed.

Local existence to problem (1.1) is also shown in [5] but in a different way and in different spaces.

2. Notation and auxiliary results. We use the anisotropic SobolevSlobodetskiu spaces $W_{2}^{l, l / 2}\left(Q^{T}\right), l \in \mathbb{R}_{+}, Q^{T}=Q \times(0, T)$, where $Q$ is either $\Omega$ (a domain in $\mathbb{R}^{3}$ ) or $S$ (the boundary of $\Omega$ ), with the norm

$$
\begin{aligned}
& \|u\|_{W_{2}^{l, l / 2}\left(Q^{T}\right)}^{2}=\sum_{|\bar{\alpha}| \leq[l]}\left\|D_{x, t}^{\bar{\alpha}} u\right\|_{L_{2}\left(Q^{T}\right)}^{2} \\
& +\sum_{|\bar{\alpha}| \leq[l]}\left(\int_{0}^{T} \int_{Q} \int_{Q} \frac{\left|D_{x, t}^{\bar{\alpha}} u(x, t)-D_{x^{\prime}, t}^{\bar{\alpha}} u\left(x^{\prime}, t\right)\right|^{2}}{\left|x-x^{\prime}\right|^{s+2(l-[l])}} d x d x^{\prime} d t\right. \\
& \left.+\int_{Q} \int_{0}^{T} \int_{0}^{T} \frac{\left|D_{x, t}^{\bar{\alpha}}(x, t)-D_{x, t^{\prime}}^{\bar{\alpha}} u\left(x, t^{\prime}\right)\right|^{2}}{\left|t-t^{\prime}\right|^{1+2(l / 2-[l / 2])}} d x d t^{\prime} d t\right) \\
& \equiv \sum_{|\bar{\alpha}| \leq[l]}\left|D_{x, t}^{\bar{\alpha}} u\right|_{2, Q^{T}}^{2} \\
& +\sum_{|\bar{\alpha}|=[l]}\left(\left[D_{x, t}^{\bar{\alpha}} u\right]_{l-[l], Q^{T}, x}^{2}+\left[D_{x, t}^{\bar{\alpha}} u\right]_{l / 2-[l / 2], Q^{T}, t}^{2}\right) \\
& \equiv\|u\|_{l, Q^{T}}^{2} \text {, }
\end{aligned}
$$


where $s=\operatorname{dim} Q, D_{x}^{\alpha}=\partial_{x_{1}}^{\alpha_{1}} \partial_{x_{2}}^{\alpha_{2}} \partial_{x_{3}}^{\alpha_{3}}, \alpha=\left(\alpha_{1}, \alpha_{2}, \alpha_{3}\right)$ is a multiindex, $|\alpha|=$ $\alpha_{1}+\alpha_{2}+\alpha_{3}, D_{x, t}^{\bar{\alpha}}=D_{x}^{\alpha} \partial_{t}^{\alpha_{0}}, \bar{\alpha}=\left(\alpha_{0}, \alpha\right), \alpha=\left(\alpha_{1}, \alpha_{2}, \alpha_{3}\right),|\bar{\alpha}|=2 \alpha_{0}+|\alpha|$ and $[l]$ is the integer part of $l$. For $Q=S$ the above norm is introduced by using local mappings and a partition of unity.

To consider problems with vanishing initial conditions we need a space of functions which admit a zero extension to $t<0$. Therefore for every $\gamma \geq 0$ we introduce the space $H_{\gamma}^{l, l / 2}\left(Q^{T}\right)$ with the norm

$$
\|u\|_{H_{\gamma}^{l, l / 2}\left(Q^{T}\right)}^{2}=\int_{0}^{T} e^{-2 \gamma t}\|u\|_{l, Q}^{2} d t+\|u\|_{H_{\gamma}^{0, l / 2}\left(Q^{T}\right)}^{2} .
$$

For $l / 2 \notin \mathbb{Z}$,

$$
\begin{aligned}
\|u\|_{H_{\gamma}^{0, l / 2}\left(Q^{T}\right)}^{2}= & \gamma^{l} \int_{0}^{T} e^{-2 \gamma t}\|u\|_{0, Q}^{2} d t \\
& +\int_{0}^{T} e^{-2 \gamma t} d t \int_{0}^{\infty} \frac{\left\|\partial_{t}^{k} u_{0}(\cdot, t-\tau)-\partial_{t}^{k} u_{0}(\cdot, t)\right\|_{0, Q}^{2}}{\tau^{1+2(l / 2-k)}} d \tau,
\end{aligned}
$$

where $k=[l / 2]<l / 2$, and $u_{0}(x, t)=u(x, t)$ for $t>0, u_{0}(x, t)=0$ for $t<0$. For $l / 2 \in \mathbb{Z}$,

$$
\|u\|_{H_{\gamma}^{0, l / 2}\left(Q^{T}\right)}^{2}=\int_{0}^{T} e^{-2 \gamma t}\left(\gamma^{l}\|u\|_{0, Q}^{2}+\left\|\partial_{t}^{l / 2} u\right\|_{0, Q}^{2}\right) d t,
$$

and we assume that $\left.\partial_{t}^{j} u\right|_{t=0}=0, j=0, \ldots, l / 2-1$, so $u_{0}(x, t)$ has a generalized derivative $\partial_{t}^{l / 2} u_{0}$ in $Q \times(-\infty, T)$. For simplicity we write $\|u\|_{l, \gamma, Q^{T}}=$ $\|u\|_{H_{\gamma}^{l, l / 2}\left(Q^{T}\right)}$. In the above definition we used the notation

$$
\|u\|_{l, Q}=\left(\sum_{|\alpha| \leq[l]}\left|D_{x}^{\alpha} u\right|_{2, Q}^{2}+\sum_{|\alpha|=[l]}\left[D_{x}^{\alpha} u\right]_{l-[l], Q, x}^{2}\right)^{1 / 2} .
$$

Set $\mathbb{R}_{+}^{n}=\left\{x \in \mathbb{R}^{n}: x_{n}>0\right\}, \mathbb{R}_{T}^{n+1}=\mathbb{R}^{n} \times(0, T), \mathbb{D}_{T}^{n+1}=\mathbb{R}_{+}^{n} \times(0, T)$, $n=2,3$. For functions defined in $\mathbb{R}_{\infty}^{n+1}$ and vanishing sufficiently fast at infinity we define the Fourier transform with respect to $x$ and the Laplace transform with respect to $t$ by the formula

$$
\widetilde{f}(\xi, s)=\int_{0}^{\infty} e^{-s t} d t \int_{\mathbb{R}^{n}} f(x, t) e^{-i x \cdot \xi} d x .
$$

Hence we define the norm

$$
\left.\left|\|u\| \|_{l, \gamma, \mathbb{R}_{\infty}^{n+1}}^{2}=\int_{\mathbb{R}^{n}} d \xi \int_{-\infty}^{\infty}\right| \widetilde{u}(\xi, s)\right|^{2}\left(|s|+|\xi|^{2}\right)^{l} d \xi_{0}, \quad s=\gamma+i \xi_{0}, \gamma \in \mathbb{R}_{+} .
$$


Similarly for functions defined in $\mathbb{D}_{\infty}^{n+1}$ we have

$$
\widetilde{f}\left(\xi, s, x_{n}\right)=\int_{0}^{\infty} e^{-s t} d t \int_{\mathbb{R}^{n-1}} f(x, t) e^{-i x^{\prime} \cdot \xi^{\prime}} d x^{\prime}, \quad x^{\prime}=\left(x_{1}, \ldots, x_{n-1}\right),
$$

and introduce the norm

$$
\begin{aligned}
\|u\|_{l, \gamma, \mathbb{D}_{\infty}^{n+1}}^{2}= & \sum_{j \leq[l]} \int_{\mathbb{R}^{n-1}} d \xi^{\prime} \int_{-\infty}^{\infty}\left\|\partial_{x_{3}}^{j} \widetilde{u}\left(\xi^{\prime}, s, x_{3}\right)\right\|_{0, \mathbb{R}_{+}^{1}}^{2}\left(|s|+\left|\xi^{\prime}\right|^{2}\right)^{l-j} d \xi_{0} \\
& +\int_{\mathbb{R}^{n-1}} d \xi^{\prime} \int_{-\infty}^{\infty}\left\|\widetilde{u}\left(\xi^{\prime}, s, \cdot\right)\right\|_{l, \mathbb{R}_{+}^{1}}^{2} d \xi_{0}, \quad s=\gamma+i \xi_{0}, \quad \gamma \in \mathbb{R}_{+} .
\end{aligned}
$$

We introduce

$$
\stackrel{\circ}{W}_{2}^{l, l / 2}\left(Q^{T}\right)=\left\{u \in W_{2}^{l, l / 2}\left(Q^{T}\right):\left.\partial_{t}^{i} u\right|_{t=0}=0, i \leq[l / 2-1 / 2]\right\}
$$

and $W_{2, \chi}^{l, l / 2}\left(Q^{T}\right)$ to be the space with the norm $\|u\|_{l, Q^{T}}+\|u\|_{[l]+\chi, Q^{T}}$, where

$$
\|u\|_{[l]+\chi, Q^{T}}=\left(\sum_{|\alpha|=[l]} \int_{0}^{T} \frac{\left|D_{x, t}^{\alpha} u\right|_{2, Q}^{2}}{t^{2 \chi}} d t\right)^{1 / 2} .
$$

For functions defined in $\Omega$ we introduce $\|u\|_{l, \Omega}=\|u\|_{H^{l}(\Omega)},|u|_{p, \Omega}=$ $\|u\|_{L_{p}(\Omega)}, l \in \mathbb{N} \cup\{0\}, 1 \leq p \in \mathbb{R}$, and we define $\Gamma_{k}^{l}(\Omega)$ to be the space with the norm

$$
\|u\|_{\Gamma_{k}^{l}(\Omega)}=\sum_{2 i \leq l-k}\left\|\partial_{t}^{i} u\right\|_{H^{l-2 i}(\Omega)} \equiv|u|_{l, k, \Omega} .
$$

Similarly we define $\Gamma_{k}^{l}(S)$.

We introduce a partition of unity. Let us define two collections of open subsets $\left\{\omega^{(k)}\right\}$ and $\left\{\Omega^{(k)}\right\}, k \in \mathfrak{M} \cup \mathfrak{N}$, such that $\bar{\omega}^{(k)} \subset \Omega^{(k)} \subset \Omega, \bigcup_{k} \omega^{(k)}=$ $\bigcup_{k} \Omega^{(k)}=\Omega, \bar{\Omega}^{(k)} \cap S=\emptyset$ for $k \in \mathfrak{M}$ and $\bar{\Omega}^{(k)} \cap S \neq \emptyset$ for $k \in \mathfrak{N}$. Assume that at most $N_{0}$ of the $\Omega^{(k)}$ have nonempty intersection. Suppose $\sup _{k} \operatorname{diam} \Omega^{(k)} \leq 2 \lambda$ for some $\lambda>0$. Let $\zeta^{(k)}(x)$ be a smooth function such that $0 \leq \zeta^{(k)}(x) \leq 1, \zeta^{(k)}(x)=1$ for $x \in \omega^{(k)}, \zeta^{(k)}(x)=0$ for $\Omega \backslash \Omega^{(k)}$ and $\left|D_{x}^{\nu} \zeta^{(k)}(x)\right| \leq c / \lambda^{|\nu|}$. Then $1 \leq \sum\left(\zeta^{(k)}(x)\right)^{2} \leq N_{0}$. Introduce the function

$$
\eta^{(k)}(x)=\frac{\zeta^{(k)}(x)}{\sum_{l}\left(\zeta^{(l)}(x)\right)^{2}} .
$$

We have $\eta^{(k)}(x)=0$ for $x \in \Omega \backslash \Omega^{(k)}, \sum_{k} \eta^{(k)}(x) \zeta^{(k)}(x)=1$ and $\left|D^{\nu} \eta^{(k)}(x)\right|$ $\leq c / \lambda^{|\nu|}$. By $\xi^{(k)}$ we denote the center of $\omega^{(k)}$ and $\Omega^{(k)}$ for $k \in \mathfrak{M}$ and the center of $\bar{\omega}^{(k)} \cap S$ and $\bar{\Omega}^{(k)} \cap S$ for $k \in \mathfrak{N}$.

Considering problems invariant with respect to translations and rotations we can introduce a local coordinate system $y=\left(y_{1}, y_{2}, y_{3}\right)$ with center at 
$\xi^{(k)}$ such that the part $\widetilde{S}^{(k)}=S \cap \bar{\Omega}^{(k)}$ of the boundary is described by $y_{3}=F\left(y_{1}, y_{2}\right)$. Then we consider new coordinates defined by

$$
z_{i}=y_{i}, \quad i=1,2, \quad z_{3}=y_{3}-F\left(y_{1}, y_{2}\right) .
$$

We will denote this transformation by $z=\Phi_{k}(y)$, where $y \in \omega^{(k)} \subset \Omega^{(k)}$; we assume that the latter sets are described in local coordinates at $\xi^{(k)}$ by the inequalities

$$
\begin{aligned}
& \left|y_{i}\right| \leq \lambda, \quad i=1,2, \quad 0<y_{3}-F\left(y_{1}, y_{2}\right) \leq \lambda \\
& \left|y_{i}\right| \leq 2 \lambda, \quad i=1,2, \quad 0<y_{3}-F\left(y_{1}, y_{2}\right) \leq 2 \lambda
\end{aligned}
$$

respectively.

Assume $S \in H^{4-1 / 2}$. Then $\|F\|_{4-1 / 2, \widetilde{S}^{(k)}} \leq M$, where $M$ can be chosen independently of $\xi \in S$. We extend $F$ to a function $\widetilde{F}$ on $\mathbb{R}_{+}^{3}$ in such a way that $\|\widetilde{F}\|_{4, \mathbb{R}_{+}^{3}} \leq c M$. Moreover, $\widetilde{F}$ satisfies $\widetilde{F}(0)=0, \nabla \widetilde{F}(0)=0$. Therefore, the following inequalities hold:

$$
|\widetilde{F}(z)| \leq c \lambda M, \quad|\nabla \widetilde{F}(z)| \leq c \lambda^{a} M, \quad a>0 .
$$

Let $y=Y_{k}(t)$ be a transformation from coordinates $x$ to local coordinates $y$ which is the composition of a translation and a rotation. Then we set

$$
\widehat{u}^{(k)}(z, t)=u\left(\Phi_{k}^{-1} \circ Y_{k}^{-1}(z), t\right), \quad \widetilde{u}^{(k)}(z, t)=\widehat{u}^{(k)}(z, t) \widehat{\zeta}^{(k)}(z, t) .
$$

Now we recall some results.

Lemma 2.1 (see [5]). Let $u \in H_{\gamma}^{r, r / 2}\left(\Omega^{T}\right)$. Then for every $\varepsilon \in(0,1)$ and $0 \leq q<r-|\alpha|$,

$$
\begin{aligned}
\left\|D_{x}^{\alpha} u\right\|_{q, \gamma, \Omega^{T}} & \leq \varepsilon^{r-|\alpha|-q}\|u\|_{r, \gamma, \Omega^{T}}+c \varepsilon^{-q-|\alpha|}\left\|e^{-\gamma t} u\right\|_{0, \Omega^{T}} \\
& \leq\left(\varepsilon^{r-|\alpha|-q}+c \gamma^{-r / 2} \varepsilon^{-q-|\alpha|}\right)\|u\|_{r, \gamma, \Omega^{T}} .
\end{aligned}
$$

Lemma 2.2 (see [5]). There exist constants $c_{1}$ and $c_{2}$, which do not depend on $u$ and $\gamma$, such that

$$
c_{1}\left|\|u\|_{l, \gamma, \mathbb{R}_{\infty}^{n+1}} \leq\|u\|_{l, \gamma, \mathbb{R}_{\gamma}^{n+1}} \leq c_{2}\right|\|u\|_{l, \gamma, \mathbb{R}_{\gamma}^{n+1}} .
$$

LEMMA 2.3 (see [5]). There exist constants $c_{3}$ and $c_{4}$, which do not depend on $u$ and $\gamma$, such that

$$
c_{3} \mid\|u\|_{l, \gamma, \mathbb{D}_{\infty}^{n+1}} \leq\|u\|_{l, \gamma, \mathbb{D}_{\gamma}^{n+1}} \leq c_{4}\|\| u \|_{l, \gamma, \mathbb{D}_{\gamma}^{n+1}} .
$$

We also need

Lemma 2.4 (see [5]). Let $u \in H_{\gamma}^{l, l / 2}\left(\mathbb{R}_{T}^{n+1}\right)$ and $0<2 m+|\alpha|<l$. Then $\partial_{t}^{m} D_{x}^{\alpha} u \in H_{\gamma}^{l_{1}, l_{1} / 2}\left(\mathbb{R}_{T}^{n+1}\right)$, where $l_{1}=l-2 m-|\alpha|$ and

$$
\left\|\partial_{t}^{m} D_{x}^{\alpha} u\right\|_{l_{1}, \gamma, \mathbb{R}_{T}^{n+1}} \leq c\|u\|_{l, \gamma, \mathbb{R}_{T}^{n+1}}
$$


Moreover, for $\varrho \in\left(0, l_{1}\right)$ and $\varepsilon>0$,

$$
\left\|\partial_{t}^{m} D_{x}^{\alpha} u\right\|_{\varrho, \gamma, \mathbb{R}_{T}^{n+1}} \leq \varepsilon^{l_{1}-\varrho}\|u\|_{l, \gamma, \mathbb{R}_{T}^{n+1}}+c \varepsilon^{-h}\left\|e^{-\gamma t} u\right\|_{0, \mathbb{R}_{T}^{n+1}},
$$

where $h=\varrho+2 m+|\alpha|$.

Let $u \in H_{\gamma}^{l, l / 2}\left(\mathbb{D}_{T}^{n+1}\right)$ and $0 \leq 2 m+|\alpha|<l-1 / 2$. Then $\left.\partial_{t}^{m} D_{x}^{\alpha} u\right|_{x_{n}=0} \in$ $H_{\gamma}^{l_{2}, l_{2} / 2}\left(\mathbb{R}_{T}^{n}\right)$, where $l_{2}=l-2 m-|\alpha|-1 / 2$, and

$$
\left\|\left.\partial_{t}^{m} D_{x}^{\alpha} u\right|_{x_{n}=0}\right\|_{l_{2}, \gamma, \mathbb{R}_{T}^{n}} \leq c\|u\|_{l, \gamma, \mathbb{D}_{T}^{n+1}} .
$$

3. Existence of solutions to problem (1.10) with vanishing initial data in the half-space. Now we consider problem (1.10) in the half-space $x_{3}>0$. First we examine the following problem:

$$
\begin{aligned}
& u_{t}-\mu \Delta u-\nu \nabla \operatorname{div} u=0, \quad x_{3}>0, \\
& \mu\left(\frac{\partial u_{i}}{\partial x_{3}}+\frac{\partial u_{3}}{\partial x_{i}}\right)=b_{i}, \quad i=1,2, \quad x_{3}=0, \\
& (\mu+\nu) \frac{\partial u_{3}}{\partial x_{3}}+(\nu-\mu)\left(\frac{\partial u_{1}}{\partial x_{1}}+\frac{\partial u_{2}}{\partial x_{2}}\right) \\
& +\sigma \Delta^{\prime} \int_{0}^{t} u_{3}(\tau) d \tau=b_{3}, \quad x_{3}=0, \\
& \left.u\right|_{t=0}=0, \quad x_{3}>0,
\end{aligned}
$$

where $\Delta^{\prime}=\partial_{x_{1}}^{2}+\partial_{x_{2}}^{2}$. By applying the Laplace-Fourier transformation

$$
\widetilde{f}\left(\xi^{\prime}, s, x_{3}\right)=\int_{0}^{\infty} e^{-s t} d t \int_{\mathbb{R}^{2}} f(x, t) e^{-i x^{\prime} \cdot \xi^{\prime}} d x^{\prime}, \quad \operatorname{Re} s>0,
$$

where $\xi^{\prime}=\left(\xi_{1}, \xi_{2}\right), x^{\prime}=\left(x_{1}, x_{2}\right), \xi^{\prime} \cdot x^{\prime}=\xi_{1} x_{1}+\xi_{2} x_{2}$, problem (3.1) takes the form

$$
\begin{aligned}
& \mu \frac{d^{2} \widetilde{u}_{k}}{d x_{3}^{2}}+\nu i \xi_{k} \frac{d \widetilde{u}_{3}}{d x_{3}}-\left(s+\mu \xi^{2}\right) \widetilde{u}_{k}-\nu \xi_{k} \xi_{j} \widetilde{u}_{j}=0, \quad k=1,2, \quad x_{3}>0, \\
& (\mu+\nu) \frac{d^{2} \widetilde{u}_{3}}{d x_{3}^{2}}+\nu i \xi_{j} \frac{d \widetilde{u}_{j}}{d x_{3}}-\left(s+\mu \xi^{2}\right) \widetilde{u}_{3}=0, \quad x_{3}=0, \\
& \mu \frac{d \widetilde{u}_{k}}{d x_{3}}+i \xi_{k} \widetilde{u}_{3}=\widetilde{b}_{k}, \quad k=1,2, \quad x_{3}=0, \\
& (\mu+\nu) \frac{d \widetilde{u}_{3}}{d x_{3}}+(\nu-\mu)\left(i \xi_{j} \widetilde{u}_{j}\right)-\frac{\sigma}{s} \xi^{2} \widetilde{u}_{3}=\widetilde{b}_{3}, \quad x_{3}=0,
\end{aligned}
$$

$\widetilde{u} \rightarrow 0$ as $x_{3} \rightarrow \infty$, where $\xi=\left(\xi_{1}, \xi_{2}\right), \xi^{2}=\xi_{1}^{2}+\xi_{2}^{2}$.

Every solution to (3.3) vanishing at infinity has the form

$$
\widetilde{u}=\Phi(\xi, s) e^{-\tau_{1} x_{3}}+\Psi(\xi, s)\left(\xi_{1}, \xi_{2}, i \tau_{2}\right) e^{-\tau_{2} x_{3}},
$$


where $\Phi(\xi, s)=\left(\phi_{1}, \phi_{2},\left(i / \tau_{1}\right) \xi \cdot \phi\right), \phi_{j}=\phi_{j}(\xi, s), j=1,2, \tau_{1}=\sqrt{s / \mu+\xi^{2}}$, $\tau_{2}=\sqrt{s /(\mu+\nu)+\xi^{2}}, \arg \tau_{j} \in(-\pi / 4, \pi / 4), j=1,2, \xi \cdot \phi=\xi_{1} \phi_{1}+\xi_{2} \phi_{2}$, $\phi=\left(\phi_{1}, \phi_{2}\right)$.

Putting (3.5) into (3.4) yields

$$
\begin{gathered}
\xi_{j} \xi \cdot \phi+\tau_{1}^{2} \phi_{j}+2 \xi_{j} \tau_{1} \tau_{2} \psi=-\mu^{-1} \widetilde{b}_{j} \tau_{1}, \quad j=1,2, \\
\left(2 \mu+\frac{\sigma}{s} \frac{\xi^{2}}{\tau_{1}}\right) \xi \cdot \phi+\left((\mu+\nu) \tau_{2}^{2}+(\mu-\nu) \xi^{2}+\frac{\sigma}{s} \xi^{2} \tau_{2}\right) \psi=i \widetilde{b}_{3} .
\end{gathered}
$$

Solving (3.6) we have

$$
\begin{aligned}
\xi \cdot \phi & =-\frac{1}{D}\left[\frac{\tau_{1}}{\mu}\left(s+2 \mu \xi^{2}+\frac{\sigma}{s} \xi^{2} \tau_{2}\right) \widetilde{b} \cdot \xi+2 \xi^{2} \tau_{1} \tau_{2} \widetilde{i}_{3}\right], \\
\psi & =\frac{1}{D}\left[\frac{\tau_{1}}{\mu}\left(2 \mu+\frac{\sigma}{s} \frac{\xi^{2}}{\tau_{1}}\right) \widetilde{b} \cdot \xi+\left(\frac{s}{\mu}+2 \xi^{2}\right) i \widetilde{b}_{3}\right]
\end{aligned}
$$

where

$$
D=\mu\left[\left(\frac{s}{\mu}+2 \xi^{2}\right)^{2}+\frac{\sigma}{\mu^{2}} \xi^{2} \tau_{2}-4 \xi^{2} \tau_{1} \tau_{2}\right] .
$$

Using (3.6) and (3.7) in (3.5) gives

$$
\begin{aligned}
\widetilde{u}_{k}= & \frac{\xi_{k}}{D} \frac{1}{\tau_{1}}\left(\tau_{1}^{2}+\xi^{2}-2 \tau_{1} \tau_{2}\right) \widetilde{b} \cdot \xi e_{1}+\frac{\xi_{k}}{D} 2\left(\tau_{1} e_{2}-\tau_{2} e_{1}\right) \widetilde{b} \cdot \xi \\
& +\frac{\xi_{k}}{D} i \widetilde{b}_{3}\left[\left(\frac{s}{\mu}+2 \xi^{2}-2 \tau_{1} \tau_{2}\right) e_{2}+2 \tau_{1} \tau_{2}\left(e_{2}-e_{1}\right)\right] \\
& +\frac{\xi_{k}}{D} \frac{\sigma}{\mu s \tau_{1}}\left(\tau_{1} e_{2}-\tau_{2} e_{1}\right) \xi^{2} \widetilde{b} \cdot \xi-\frac{1}{\mu \tau_{1}} \widetilde{b}_{k} e_{1}, \quad k=1,2, \\
\widetilde{u}_{3}= & \frac{i}{D}\left[2 \tau_{1} \tau_{2}\left(\tau_{2}-\tau_{1}\right) e_{0}+\left(2 \tau_{1} \tau_{2}-\left(\frac{s}{\mu}+2 \xi^{2}\right)\right) e_{1}\right] \widetilde{b} \cdot \xi \\
& -\frac{i}{D} \frac{\sigma}{\mu s} \xi^{2} \tau_{2}\left(\tau_{1}-\tau_{2}\right) \widetilde{b} \cdot \xi e_{0} \\
& -\frac{\tau_{2}}{D}\left[\left(\frac{s}{\mu}+2 \xi^{2}\right)\left(\tau_{2}-\tau_{1}\right) e_{0}+\frac{s}{\mu} e_{1}\right] \widetilde{b}_{3},
\end{aligned}
$$

where $e_{i}=e^{-\tau_{i} x_{3}}, i=1,2, e_{0}=\left(e_{1}-e_{2}\right) /\left(\tau_{1}-\tau_{2}\right)$.

Using the expressions

$$
\begin{gathered}
\tau_{1}^{2}-\tau_{2}^{2}=\frac{s}{\mu}-\frac{s}{\mu+\nu}=\frac{\nu}{\mu(\mu+\nu)} s \equiv c_{0} s \\
\tau_{1}^{2}+\xi^{2}-2 \tau_{1} \tau_{2}=\left(\tau_{1}-\tau_{2}\right)^{2}-\frac{s}{\mu+\nu}=\frac{c_{0} s}{\left(\tau_{1}+\tau_{2}\right)^{2}}-\frac{s}{\mu+\nu} \equiv c_{1} s, \\
\tau_{1} e_{2}-\tau_{2} e_{1}=\left(\tau_{1}-\tau_{2}\right) e_{2}+\tau_{2}\left(e_{2}-e_{1}\right)=\frac{c_{0} s}{\tau_{1}+\tau_{2}} e_{2}-\tau_{2} \frac{c_{0} s}{\tau_{1}+\tau_{2}} e_{0}
\end{gathered}
$$


we write (3.9) in the form

$$
\begin{aligned}
\widetilde{u}_{k}= & \left(\frac{\xi_{k}}{D} \frac{c_{1} s}{\tau_{1}} \widetilde{b} \cdot \xi-\frac{1}{\mu \tau_{1}} \widetilde{b}_{k}\right) e_{1} \\
& +2 \frac{\xi_{k}}{D}\left(\frac{c_{0} s}{\tau_{1}+\tau_{2}} \widetilde{b} \cdot \xi+c_{1} s i \widetilde{b}_{3}+\frac{\sigma}{\mu \tau_{1}} \frac{c_{0} \xi^{2}}{\tau_{1}+\tau_{2}} \widetilde{b} \cdot \xi\right) e_{2} \\
& -\frac{\xi_{k}}{D}\left(2 \tau_{2} \frac{c_{0} s}{\tau_{1}+\tau_{2}} \widetilde{b} \cdot \xi+2 \tau_{1} \tau_{2} \frac{c_{0} s}{\tau_{1}+\tau_{2}} \widetilde{i b}_{3}\right. \\
& \left.+\frac{\sigma}{\mu \tau_{1}} \frac{\tau_{2} c_{0}}{\tau_{1}+\tau_{2}} \xi^{2} \widetilde{b} \cdot \xi\right) e_{0} \\
\equiv & E_{k 1} e_{1}+E_{k 2} e_{2}+E_{k 0} e_{0}, \quad k=1,2, \\
\widetilde{u}_{3}= & -\frac{1}{D}\left[\left(2 \tau_{1} \tau_{2} i \frac{c_{0} s}{\tau_{1}+\tau_{2}}+\frac{i \sigma}{\mu} \frac{\xi^{2} \tau_{2} c_{0}}{\tau_{1}+\tau_{2}}\right) \widetilde{b} \cdot \xi\right. \\
& \left.+\frac{\tau_{2} c_{0} s}{\tau_{1}+\tau_{2}}\left(\frac{s}{\mu}+2 \xi^{2}\right) \widetilde{b}_{3}\right] e_{0} \\
& +\frac{1}{D}\left(i c_{1} s \widetilde{b} \cdot \xi-\frac{\tau_{2} s}{\mu} \widetilde{b}_{3}\right) e_{1} \equiv E_{30} e_{0}+E_{31} e_{1} .
\end{aligned}
$$

From $[8,12]$ we have

Lemma 3.1. For all $\xi=\left(\xi_{1}, \xi_{2}\right) \in \mathbb{R}^{2}$ and $s=\gamma+i \xi_{0}$ with $\gamma>0, \gamma \in \mathbb{R}$, $\xi_{0} \in \mathbb{R}^{1}$,

$$
|D| \geq c_{2}|s| \xi^{2}, \quad|D| \geq c_{3}\left(|s|^{2}+|\xi|^{3}\right) .
$$

Using Lemma 3.1 we obtain

Lemma 3.2. For $\xi \in \mathbb{R}^{2}$ and $\gamma=\operatorname{Re} s>0$,

$$
\left|\bar{E}_{0}\right| \leq c_{4}|\widetilde{b}|, \quad\left|\bar{E}_{1}\right|+\left|\bar{E}_{2}\right| \leq \frac{c_{5}}{\sqrt{|s|+\xi^{2}}}|\widetilde{b}|,
$$

where $\bar{E}_{i}=\left(E_{1 i}, E_{2 i}, E_{3 i}\right), i=0,1,2$.

Moreover, from [12] (see also [5]) we have

Lemma 3.3. For $\xi \in \mathbb{R}^{2}, s=\gamma+i \gamma_{0}, \gamma, \xi_{0} \in \mathbb{R}, \gamma>0$, and for every nonnegative integer $j$,

$$
\begin{aligned}
& \int_{0}^{\infty}\left|\frac{d^{j} e_{i}\left(x_{3}\right)}{d x_{3}^{j}}\right|^{2} d x_{3} \leq \frac{1}{\sqrt{2}}\left|\tau_{i}\right|^{2 j-1}, \quad i=1,2, \\
& \int_{0}^{\infty}\left|\frac{d^{j} e_{0}\left(x_{3}\right)}{d x_{3}^{j}}\right|^{2} d x_{3} \leq c \frac{\left|\tau_{1}\right|^{2 j-1}+\left|\tau_{2}\right|^{2 j-1}}{\left|\tau_{1}\right|^{2}} .
\end{aligned}
$$

Lemmas 3.1-3.3 and [12] imply 
THEOREM 3.4. Let $b_{1}, b_{2} \in H_{\gamma}^{2+1 / 2,1+1 / 4}\left(\mathbb{R}_{\infty}^{3}\right), b_{3}=d_{1}+\sigma \int_{0}^{t} d_{2}(\tau) d \tau$, $d_{1} \in H_{\gamma}^{2+1 / 2,1+1 / 4}\left(\mathbb{R}_{\infty}^{3}\right)$, and $d_{2} \in H_{\gamma}^{2-1 / 2,1-1 / 4}\left(\mathbb{R}_{\infty}^{3}\right)$. Then solutions of problem (3.1) satisfy the estimate

$$
\begin{aligned}
& \sum_{i=1}^{3}\left\|u_{i}\right\|_{4, \gamma, \mathbb{D}}{ }_{\infty}^{4} \\
& \quad \leq c(\gamma)\left(\sum_{\alpha=1}^{2}\left\|b_{\alpha}\right\|_{2+1 / 2, \gamma, \mathbb{R}_{\infty}^{3}}+\left\|d_{1}\right\|_{2+1 / 2, \gamma, \mathbb{R}_{\infty}^{3}}+\left\|d_{2}\right\|_{2-1 / 2, \gamma, \mathbb{R}_{\infty}^{3}}\right),
\end{aligned}
$$

where $c(\gamma)$ remains bounded for $\gamma>\gamma_{0}>0$.

Now we consider the problem

$$
\begin{array}{ll}
u_{t}-\mu \Delta u-\nu \nabla \operatorname{div} u=f, & x_{3}>0, \\
\mu\left(\frac{\partial u_{i}}{\partial x_{3}}+\frac{\partial u_{3}}{\partial x_{i}}\right)=b_{i}, \quad i=1,2, & x_{3}=0, \\
(\mu+\nu) \frac{\partial u_{3}}{\partial x_{3}}+(\nu-\mu)\left(\frac{\partial u_{1}}{\partial x_{1}}+\frac{\partial u_{2}}{\partial x_{2}}\right) & \\
+\sigma \Delta^{\prime} \int_{0}^{t} u_{3}(\tau) d \tau=b_{3}, & x_{3}=0, \\
\left.u\right|_{t=0}=0, & x_{3}>0 .
\end{array}
$$

In view of the considerations in [12] and Theorem 3.4 we have

THEOREM 3.5. Let the assumptions of Theorem 3.4 be satisfied. Let $f \in$ $H_{\gamma}^{2,1}\left(\mathbb{D}_{\infty}^{4}\right)$. Then there exists a solution to (3.14) such that $u \in H_{\gamma}^{4,2}\left(\mathbb{D}_{\infty}^{4}\right)$ and

$$
\begin{aligned}
(3.15) \sum_{i=1}^{3}\left\|u_{i}\right\|_{4, \gamma, \mathbb{D}_{\infty}^{4}} \leq & c(\gamma)\left(\sum_{\alpha=1}^{2}\left\|b_{\alpha}\right\|_{2+1 / 2, \gamma, \mathbb{R}_{\infty}^{3}}+\left\|d_{1}\right\|_{2+1 / 2, \gamma, \mathbb{R}_{\infty}^{3}}\right. \\
& \left.+\left\|d_{2}\right\|_{2-1 / 2, \gamma, \mathbb{R}_{\infty}^{3}}+\|f\|_{2, \gamma, \mathbb{D}_{\infty}^{4}}\right) .
\end{aligned}
$$

4. Existence of solutions to problem (1.10). First we consider problem (1.10) with vanishing initial data:

$$
\begin{array}{rlrl}
L\left(\partial_{x}, \partial_{t}\right) & \equiv u_{t}-\mu \Delta u-\nu \nabla \operatorname{div} u=f & & \text { in } \Omega \times(-\infty, T), \\
B_{1}\left(x, \partial_{x}\right) u & \equiv \Pi_{0} \mathbb{T}(u) \bar{n}_{0}=\Pi_{0} b \equiv b^{\prime} & & \text { on } S \times(-\infty, T), \\
B_{2}\left(x, \partial_{x}\right) u & \equiv \bar{n}_{0} \mathbb{T}(u) \bar{n}_{0}-\sigma \bar{n}_{0} \Delta_{S} \int_{0}^{t} u(\tau) d \tau=b \cdot \bar{n} & \\
& \equiv d_{1}+\sigma \int_{0}^{t} d_{2}(\tau) d \tau & & \text { on } S \times(-\infty, T),
\end{array}
$$


where $\Pi_{0} b=b-\left(b \cdot \bar{n}_{0}\right) \bar{n}_{0} \equiv b^{\prime}$. We write $B\left(x, \partial_{x}\right) u=\left(B_{1}\left(x, \partial_{x}\right) u\right.$, $\left.B_{2}\left(x, \partial_{x}\right) u\right)$.

Let $f^{(k)}(x, t)=\zeta^{(k)}(x, t) f(x, t)$. We denote by $R^{(k)}, k \in \mathfrak{M}$, the operator

$$
u^{(k)}(x, t)=R^{(k)} f^{(k)}(x, t),
$$

where $u^{(k)}(x, t)$ is the solution of the Cauchy problem

$$
L\left(\partial_{x}, \partial_{t}\right) u^{(k)}(x, t)=f^{(k)}(x, t) .
$$

For $k \in \mathfrak{N}$ we define $R^{(k)}$ to be the operator

$$
\widehat{u}^{(k)}(z, t)=R^{(k)}\left(\widehat{f}^{(k)}(z, t), \widehat{b}^{(k)}(z, t)\right),
$$

where $\widehat{u}^{(k)}(z, t)$ is the solution to the boundary value problem

$$
L\left(\partial_{z}, \partial_{t}\right) \widehat{u}^{(k)}(z, t)=\widehat{f}^{(k)}(z, t), \quad B\left(z, \partial_{z}\right) \widehat{u}^{(k)}(z, t)=\widehat{b}^{(k)}(z, t),
$$

where $\widehat{u}^{(k)}(z, t)=Z_{k}^{-1} u^{(k)}(x, t)$ and $Z_{k}$ is the operator which represents the relation between $\widehat{u}^{(k)}(z, t)$ and $u^{(k)}(x, t)$.

Then we define an operator $R$ (called a regularizer) by the formula (see $[3,6])$

$$
R h=\sum_{k} \eta^{(k)}(x) u^{(k)}(x, t)
$$

where

and

$$
h^{(k)}(x, t)= \begin{cases}f^{(k)}(x, t), & k \in \mathfrak{M} \\ \left\{\widehat{f}^{(k)}(z, t), \widehat{b}^{(k)}(z, t)\right\}, & k \in \mathfrak{N}\end{cases}
$$

$$
u^{(k)}(x, t)= \begin{cases}R^{(k)} f^{(k)}(x, t), & k \in \mathfrak{M}, \\ Z_{k} R^{(k)}\left(Z_{k}^{-1} f^{(k)}(x, t), Z_{k}^{-1} b^{(k)}(x, t)\right), & k \in \mathfrak{N} .\end{cases}
$$

Theorem 3.5 implies existence of solutions of problems (4.3), (4.5) and the estimates

$$
\left\|u^{(k)}\right\|_{4, \gamma, \mathbb{R}_{\infty}^{4}} \leq c\left\|f^{(k)}\right\|_{2, \gamma, \mathbb{D}_{\infty}^{4}}, \quad k \in \mathfrak{M},
$$

and

(4.8) $\left\|\widehat{u}^{(k)}\right\|_{4, \gamma, \mathbb{R}_{\infty}^{4}} \leq c\left(\left\|\widehat{f}^{(k)}\right\|_{2, \gamma, \mathbb{D}_{\infty}^{4}}+\sum_{i=1}^{2}\left\|\widehat{b}_{i}^{(k)}\right\|_{2+1 / 2, \gamma, \mathbb{R}_{\infty}^{3}}\right.$

$$
\left.+\left\|\widehat{d}_{1}^{(k)}\right\|_{2+1 / 2, \gamma, \mathbb{R}_{\infty}^{3}}+\left\|\widehat{d}_{2}^{(k)}\right\|_{2-1 / 2, \gamma, \mathbb{R}_{\infty}^{3}}\right), \quad k \in \mathfrak{N} .
$$

Let $h=\left(f, b_{1}, b_{2}, d_{1}, d_{2}\right) \in H_{\gamma}^{l, l / 2}\left(\Omega^{T}\right) \times H_{\gamma}^{l+1 / 2, l / 2+1 / 4}\left(S^{T}\right) \times$ $H_{\gamma}^{l+1 / 2, l / 2+1 / 4}\left(S^{T}\right) \times H_{\gamma}^{l+1 / 2, l / 2+1 / 4}\left(S^{T}\right) \times H_{\gamma}^{l-1 / 2, l / 2-1 / 4}\left(S^{T}\right) \equiv H_{\gamma}^{l}$ and let $V_{\gamma}^{l}=H_{\gamma}^{l+2, l / 2+1}\left(\Omega^{T}\right)$.

Inequalities (4.7) and (4.8) imply 
LEMmA 4.1 (see [12]). Let $S \in H^{4-1 / 2}$ and $h \in H_{\gamma}^{2}$ with $\gamma$ sufficiently large. Then there exists a bounded linear operator $R: H_{\gamma}^{2} \rightarrow V_{\gamma}^{2}$ such that

$$
\|R h\|_{V_{\gamma}^{2}} \leq c\|h\|_{H_{\gamma}^{2}}
$$

where $c$ does not depend on $\gamma$ and $h$.

We write problem (4.1) in the following short form:

$$
A u=h, \quad A=(L, B) .
$$

Lemma 4.2. Let $S \in H^{4-1 / 2}$ and $h \in H_{\gamma}^{2}$ with $\gamma$ sufficiently large. Then

$$
A R h=h+T h,
$$

where $T$ is a bounded operator in $H_{\gamma}^{2}$ with small norm for small $\lambda$ and large $\gamma$.

Proof. We have

$$
\begin{aligned}
L R h= & \sum_{k \in \mathfrak{M} \cup \mathfrak{N}}\left(L\left(\partial_{x}, \partial_{t}\right) \eta^{(k)} u^{(k)}-\eta^{(k)} L\left(\partial_{x}, \partial_{t}\right) u^{(k)}\right) \\
& +\sum_{k \in \mathfrak{N}} \eta^{(k)} Z_{k}\left(L\left(\partial_{z}-\nabla F \partial_{z_{3}}, \partial_{t}\right)-L\left(\partial_{z}, \partial_{t}\right)\right) Z_{k}^{-1} u^{(k)}(x, t) \\
& +\sum_{k \in \mathfrak{M}} \eta^{(k)} L\left(\partial_{x}, \partial_{t}\right) u^{(k)}(x, t)+\sum_{k \in \mathfrak{N}} \eta^{(k)} Z_{k} L\left(\partial_{z}, \partial_{t}\right) Z_{k}^{-1} u^{(k)}(x, t) \\
= & f+T_{1} h,
\end{aligned}
$$

and

$$
\begin{aligned}
B R h= & \sum_{k \in \mathfrak{M} \cup \mathfrak{N}}\left(B\left(x, \partial_{x}\right) \eta^{(k)} u^{(k)}-\eta^{(k)} B\left(x, \partial_{x}\right) u^{(k)}\right) \\
& +\sum_{k \in \mathfrak{M} \cup \mathfrak{N}} \eta^{(k)}\left(B\left(x, \partial_{x}\right)-B\left(\xi^{(k)}, \partial_{x}\right)\right) u^{(k)}+\sum_{k \in \mathfrak{M}} \eta^{(k)} b^{(k)} \\
& +\sum_{k \in \mathfrak{N}} \eta^{(k)} Z_{k}\left(B\left(\xi^{(k)}, \partial_{z}-\nabla F \partial_{z_{3}}\right)-B\left(\xi^{(k)}, \partial_{z}\right)\right) Z^{-1} u^{(k)}(x, t) \\
& +\sum_{k \in \mathfrak{N}} \eta^{(k)} Z_{k} B\left(\xi^{(k)}, \partial_{z}\right) Z_{k}^{-1} u^{(k)}(x, t)=b+T_{2} h .
\end{aligned}
$$

Now we estimate operators $T_{1}$ and $T_{2}$. By using Lemmas 2.1, 2.4 and Theorems 3.4, 3.5 the first term in $T_{1} h$ is estimated in the following way:

$$
\begin{aligned}
& \left\|\sum_{k \in \mathfrak{M} \cup \mathfrak{N}}\left(L \eta^{(k)} u^{(k)}-\eta^{(k)} L u^{(k)}\right)\right\|_{2, \gamma, \Omega^{T}} \leq c \sum_{k \in \mathfrak{M} \cup \mathfrak{N}}\left\|u^{(k)}\right\|_{3, \gamma, Q^{(k)}} \\
& \quad \leq c\left(\varepsilon^{\delta_{1}}+c_{0}(\varepsilon) \gamma^{-\delta_{2}}\right) \sum_{k \in \mathfrak{M} \cup \mathfrak{N}}\left\|u^{(k)}\right\|_{4, \gamma, Q^{(k)}} \leq c\left(\varepsilon^{\delta_{1}}+c_{0}(\varepsilon) \gamma^{-\delta_{2}}\right)\|h\|_{H_{\gamma}^{2}},
\end{aligned}
$$

where $\delta_{i}>0, i=1,2, Q^{(k)}=\Omega^{(k)} \times(0, T)$, and $c_{0}(\varepsilon)$ is a decreasing function. 
The second term in $T_{1} h$ is bounded by

$$
\begin{aligned}
c \sum_{k \in \mathfrak{N}}\left(\|\left.\left(\nabla \widetilde{F} \nabla^{2} \widetilde{F} \nabla \widehat{u}^{(k)}\right)\right|_{z}=\Phi_{k}(y(x))\right. & \|_{2, \gamma, Q^{(k)}} \\
& +\left\|\left.\left(\nabla \widetilde{F}(1+\nabla \widetilde{F}) \nabla^{2} \widehat{u}^{(k)}\right)\right|_{z=\Phi_{k}(y(x))}\right\|_{2, \gamma, Q^{(k)}} \\
& \left.+\left\|\left.\left(\nabla^{2} \widetilde{F} \nabla \widehat{u}^{(k)}\right)\right|_{z=\Phi_{k}(y(x))}\right\|_{2, \gamma, Q^{(k)}}\right) \\
\leq & c \sum_{k \in \mathfrak{N}}\left(p\left(\|\nabla \widetilde{F}\|_{3, Q^{(k)}}\right)\left\|u^{(k)}\right\|_{3, \gamma, Q^{(k)}}\right. \\
& \left.+\sup _{Q^{(k)}}|\nabla \widetilde{F}|\left(1+\sup _{Q^{(k)}}|\nabla \widetilde{F}|\right)\left\|u^{(k)}\right\|_{4, \gamma, Q^{(k)}}\right) \equiv I,
\end{aligned}
$$

where $p$ is a polynomial of degree two. Using $\sup _{\Omega^{(k)}}|\nabla \widetilde{F}| \leq c \lambda^{1 / 2}\|\nabla \widetilde{F}\|_{3, \Omega^{(k)}}$, the interpolation inequalities and Theorems 3.4 and 3.5 we have

$$
I \leq c\left(\varepsilon^{\delta_{1}}+c_{0}(\varepsilon)\left(\lambda^{\delta_{2}}+\gamma^{-\delta_{3}}\right)\right)\|h\|_{H_{\gamma}^{2}}, \quad \delta_{i}>0, i=1,2,3,
$$

and $c_{0}(\varepsilon)$ is a decreasing function.

Similar considerations can be applied to the other terms of $T_{1}$ and $T_{2}$. Summarizing we have

$$
\|T h\|_{H_{\gamma}^{2}} \leq c\left[\varepsilon^{\delta_{1}}+c_{0}(\varepsilon)\left(\lambda^{d_{2}}+\gamma^{-\delta_{3}}\right)\right]\|h\|_{H_{\gamma}^{2}} .
$$

This concludes the proof.

Lemma 4.2 (see [12]). Let $S \in H^{4-1 / 2}$. Then for every $v \in V_{\gamma}^{2}$,

$$
R A v=v+W v,
$$

where $W$ is a bounded operator in $V_{\gamma}^{2}$ whose norm can be made small for small $\lambda$ and large $\gamma$, because

$$
\|W v\|_{V_{\gamma}^{2}} \leq c\left[\varepsilon^{\delta_{1}}+c_{0}(\varepsilon)\left(\lambda^{\delta_{2}}+\gamma^{-\delta_{3}}\right)\right]\|v\|_{V_{\gamma}^{2}}, \quad \varepsilon \in(0,1),
$$

where $c_{0}(\varepsilon)$ is a decreasing function.

Proof. See the proof of Theorem 3.4 of [12].

For sufficiently large $\gamma$ and sufficiently small $\varepsilon$ and $\lambda$ the norms of $W$ and $T$ are less than one. Therefore Lemmas 4.1 and $4.2 \mathrm{imply}$

Theorem 4.3. Let $f \in H_{\gamma}^{2,1}\left(\Omega^{T}\right), b^{\prime}, d_{1} \in H_{\gamma}^{2+1 / 2,1+1 / 4}\left(S^{T}\right), d_{2} \in$ $H_{\gamma}^{2-1 / 2,1-1 / 4}\left(S^{T}\right)$ and $S \in H_{\gamma}^{4-1 / 2}$. Then for sufficiently large $\gamma$ there exists a unique solution of problem (4.1) such that $u \in H_{\gamma}^{4,2}\left(\Omega^{T}\right)$ and

$$
\begin{aligned}
\|u\|_{4, \gamma, \Omega^{T}} \leq & c\left(\|f\|_{2, \gamma, \Omega^{T}}+\left\|b^{\prime}\right\|_{2+1 / 2, \gamma, S^{T}}\right. \\
& \left.+\left\|d_{1}\right\|_{2+1 / 2, \gamma, S^{T}}+\left\|d_{2}\right\|_{2-1 / 2, \gamma, S^{T}}\right),
\end{aligned}
$$

where $c$ does not depend on $u$ and $\gamma$. 
Now we consider problem (1.10) with nonvanishing initial data. Then we have

TheOrEm 4.4. Let $f \in W_{2}^{2,1}\left(\Omega^{T}\right), g_{i} \in W_{2,1 / 4}^{2+1 / 2,1+1 / 4}\left(S^{T}\right), i=1,2$, $h \in W_{2}^{1+1 / 2,1 / 2+1 / 4}\left(S^{T}\right), S \in H^{4-1 / 2}, u_{0} \in H^{3}(\Omega)$, and $T<\infty$. Then there exists a solution of problem (1.10) such that $u \in W_{2}^{4,2}\left(\Omega^{T}\right)$ and

$$
\|u\|_{4, \Omega^{T}} \leq c(T)\left(X_{1}+X_{2}\right)
$$

where $X_{1}=\left\|f_{1}\right\|_{2, \Omega^{T}}+\sum_{i=1}^{2}\left\|g_{i}\right\|_{(2+1 / 2), S^{T}, 1 / 4}+\|h\|_{1+1 / 2, S^{T}}, X_{2}=$ $\|f(0)\|_{1, \Omega}+\left\|u_{0}\right\|_{3, \Omega}$, and $c(T)$ is an increasing function of $T$.

Proof. Let $\phi^{0}=u_{0} \in H^{3}(\Omega)$ and $\phi^{1}=\mu \Delta u_{0}+\nu \nabla \operatorname{div} u_{0}+f(0) \in$ $H^{1}(\Omega)$. We extend the functions onto $\mathbb{R}^{3}$ in such a way that the extended functions $\widetilde{\phi}^{0}, \widetilde{\phi}^{1}$ satisfy $\widetilde{\phi}^{0} \in H^{3}\left(\mathbb{R}^{3}\right), \widetilde{\phi}^{1} \in H^{1}\left(\mathbb{R}^{3}\right)$ and $\left\|\widetilde{\phi}^{0}\right\|_{3, \mathbb{R}^{3}} \leq c\left\|\phi^{0}\right\|_{3, \Omega}$, $\left\|\widetilde{\phi}^{1}\right\|_{1, \mathbb{R}^{3}} \leq c\left\|\phi^{1}\right\|_{1, \Omega}$.

In view of Lemma 4.5 below there exists a function $\widetilde{v} \in W_{2}^{4,2}\left(\mathbb{R}^{3} \times \mathbb{R}^{1}\right)$ such that

$$
\left.\partial_{t}^{i} \widetilde{v}\right|_{t=0}=\widetilde{\phi}^{i}, \quad i=1,2
$$

and

$$
\begin{aligned}
\|v\|_{4, \Omega^{T}} & \leq\|\widetilde{v}\|_{4, \mathbb{R}^{3} \times \mathbb{R}^{1}} \leq c\left(\left\|\widetilde{\phi}^{0}\right\|_{3, \mathbb{R}^{3}}+\left\|\widetilde{\phi}^{1}\right\|_{1, \mathbb{R}^{3}}\right) \\
& \leq c\left(\left\|u_{0}\right\|_{3, \Omega}+\|f(0)\|_{1, \Omega}\right),
\end{aligned}
$$

where $v=\left.\widetilde{v}\right|_{\Omega^{T}}$. Introducing the function

$$
w=u-v
$$

we see that it is a solution of the problem

$$
\begin{array}{ll}
w_{t}-\mu \Delta w-\nu \nabla \operatorname{div} w=f^{\prime} & \text { in } \Omega^{T}, \\
\Pi_{0} \mathbb{D}(w) \bar{n}_{0}=g_{1}^{\prime} & \text { on } S^{T}, \\
\bar{n}_{0} \mathbb{D}(w) \bar{n}_{0}-\sigma \bar{n}_{0} \Delta_{S}(0) \int_{0}^{t} w(\tau) d \tau=g_{2}^{\prime}+\sigma \int_{0}^{t} h^{\prime}(\tau) d \tau & \text { on } S^{T}, \\
\left.w\right|_{t=0}=0 & \text { in } \Omega,
\end{array}
$$

where

$$
\begin{aligned}
& f^{\prime}=f-\left(v_{t}-\mu \Delta v-\nu \nabla \operatorname{div} v\right) \in \stackrel{\circ}{W}_{2}^{2,1}\left(\Omega^{T}\right), \\
& g_{1}^{\prime}=g_{1}-\Pi_{0} \mathbb{D}(v) \bar{n}_{0} \in \stackrel{\circ}{W}_{2}^{2+1 / 2,1+1 / 4}\left(S^{T}\right), \\
& g_{2}^{\prime}=g_{2}-\bar{n}_{0} \mathbb{D}(v) \bar{n} \in \stackrel{\circ}{W}_{2}^{2+1 / 2,1+1 / 4}\left(S^{T}\right), \\
& h^{\prime}=h-\bar{n}_{0} \Delta_{S}(0) v \in \stackrel{\circ}{W}_{2}^{2-1 / 2,1-1 / 4}\left(S^{T}\right) .
\end{aligned}
$$

To prove existence of solutions to problem (4.20) we have to extend the right-hand side functions by zero for $t<0$. The function $f^{\prime}$ can be extended 
easily to a function $f^{\prime \prime} \in H_{0}^{2,1}\left(\Omega^{T}\right)$ and

$$
\left\|f^{\prime \prime}\right\|_{2,0, \Omega^{T}} \leq c\left\|f^{\prime}\right\|_{2, \Omega^{T}} \leq c\left(\|f\|_{2, \Omega^{T}}+\|v\|_{4, \Omega^{T}}\right) .
$$

Since $1-1 / 4-[1-1 / 4]=3 / 4>1 / 2$, in view of Lemma 2.5 of [13], $h^{\prime}$ can be extended by zero to a function $h^{\prime \prime} \in H_{0}^{2-1 / 2,1-1 / 4}\left(S^{T}\right)$ and

$$
\left\|h^{\prime \prime}\right\|_{2-1 / 2,0, S^{T}} \leq c\left\|h^{\prime}\right\|_{2-1 / 2, S^{T}} \leq c\left(\|h\|_{2-1 / 2, S^{T}}+\|v\|_{4, \Omega^{T}}\right) .
$$

Since $1+1 / 4-[1+1 / 4]=1 / 4<1 / 2$, to extend the function $g_{i}^{\prime}, i=$ 1,2 , we have to assume that $g_{i}^{\prime} \in W_{2,1 / 4}^{2+1 / 2,1+1 / 4}\left(S^{T}\right), i=1,2$. Hence $g_{i} \in$ $H_{2,1 / 4}^{2+1 / 2,1+1 / 4}\left(S^{T}\right), i=1,2$, and $v$ must be such that

$$
\begin{aligned}
\left\|\Pi_{0} \mathbb{D}(v) \bar{n}_{0}\right\|_{(2+1 / 2), S^{T}, 1 / 4}+\left\|\bar{n}_{0} \mathbb{D}(v) \bar{n}_{0}\right\|_{(2+1 / 2), S^{T}, 1 / 4} \\
\leq c\left(\left\|u_{0}\right\|_{3, \Omega}+\|f(0)\|_{1, \Omega}\right) .
\end{aligned}
$$

If we show this, then the extended functions $g_{i}^{\prime \prime} \in H_{0}^{2+1 / 2,1+1 / 4}\left(S^{T}\right), i=1,2$, and

$$
\begin{aligned}
\left\|g_{i}^{\prime \prime}\right\|_{2+1 / 2,0, S^{T}} & \leq c\left\|g_{i}^{\prime}\right\|_{(2+1 / 2), S^{T}, 1 / 4} \\
& \leq c\left(\left\|g_{i}\right\|_{(2+1 / 2), S^{T}, 1 / 4}+\left\|u_{0}\right\|_{3, \Omega}+\|f(0)\|_{1, \Omega}\right) .
\end{aligned}
$$

To prove (4.24) it is sufficient to estimate the expressions

$$
\begin{gathered}
\left(\int_{0}^{T} \frac{\left|D_{\xi, t}^{2}\left(\Pi_{0} \mathbb{D}_{\xi}(v) \bar{n}_{0}\right)\right|_{2, S}^{2}}{t^{1 / 2}} d t\right)^{1 / 2}+\left(\int_{0}^{T} \frac{\left|D_{\xi, t}^{2}\left(\bar{n}_{0} \mathbb{D}(v) \bar{n}_{0}\right)\right|_{2, S}^{2}}{t^{1 / 2}} d t\right)^{1 / 2} \\
\leq\left(\int_{0}^{T} \frac{\left|D_{\xi} v\right|_{2, S}^{2}+\left|D_{\xi}^{2} v\right|_{2, S}^{2}+\left|D_{\xi}^{3} v\right|_{2, S}^{2}+\left|D_{\xi} \partial_{t} v\right|_{2, S}^{2}}{t^{1 / 2}} d t\right)^{1 / 2} \\
\quad \leq\left(\int_{0}^{\infty} \frac{\left|D_{\xi} \widetilde{v}\right|_{2, S}^{2}+\left|D_{\xi}^{2} \widetilde{v}\right|_{2, S}^{2}+\left|D_{\xi}^{3} \widetilde{v}\right|_{2, S}^{2}+\left|D_{\xi} \partial_{t} \widetilde{v}\right|_{2, S}^{2}}{t^{1 / 2}} d t\right)^{1 / 2} \equiv I
\end{gathered}
$$

where we have used the fact that $S \in H^{4-1 / 2}$. In view of Lemma 2.6 of [13] we have the estimate

$$
\begin{aligned}
I \leq & c\left(\int _ { 0 } ^ { \infty } d t \int _ { 0 } ^ { \infty } d t ^ { \prime } \left(\frac{\left|D_{\xi} \widetilde{v}(t)-D_{\xi} \widetilde{v}\left(t^{\prime}\right)\right|_{2, S}^{2}}{\left|t-t^{\prime}\right|^{1+1 / 2}}+\frac{\left|D_{\xi}^{2} \widetilde{v}(t)-D_{\xi}^{2} \widetilde{v}\left(t^{\prime}\right)\right|_{2, S}^{2}}{\left|t-t^{\prime}\right|^{1+1 / 2}}\right.\right. \\
& \left.\left.+\frac{\left|D_{\xi}^{3} \widetilde{v}(t)-D_{\xi}^{3} \widetilde{v}\left(t^{\prime}\right)\right|_{2, S}^{2}}{\left|t-t^{\prime}\right|^{1+1 / 2}}+\frac{\left|D_{\xi} \partial_{t} \widetilde{v}(t)-D_{\xi} \partial_{t^{\prime}} \widetilde{v}\left(t^{\prime}\right)\right|_{2, S}^{2}}{\left|t-t^{\prime}\right|^{1+1 / 2}}\right)\right)^{1 / 2}
\end{aligned}
$$

$\leq c\|\widetilde{v}\|_{4, \Omega \times \mathbb{R}^{1}}$.

Hence in view of (4.18) we have (4.24).

Since $T<\infty$ the norms of $H_{\gamma}^{l, l / 2}\left(\Omega^{T}\right)$ and $H_{0}^{l, l / 2}\left(\Omega^{T}\right)$ are equivalent (and similarly for boundary norms). Therefore, $f^{\prime \prime} \in H_{\gamma}^{2,1}\left(\Omega^{T}\right), g_{1}^{\prime \prime}, g_{2}^{\prime \prime} \in$ 
$H_{\gamma}^{2+1 / 2,1+1 / 4}\left(S^{T}\right), h^{\prime \prime} \in H_{\gamma}^{1+1 / 2,1 / 2+1 / 4}\left(S^{T}\right)$ and there exists a constant $c(\gamma)$ such that

$$
\begin{aligned}
& \left\|f^{\prime \prime}\right\|_{2, \gamma, \Omega^{T}} \leq c(\gamma)\left\|f^{\prime \prime}\right\|_{2,0, \Omega^{T}}, \\
& \left\|g_{i}^{\prime \prime}\right\|_{2+1 / 2, \gamma, S^{T}} \leq c(\gamma)\left\|g_{i}^{\prime \prime}\right\|_{2+1 / 2,0, S^{T}}, \quad i=1,2, \\
& \left\|h^{\prime \prime}\right\|_{1+1 / 2, \gamma, S^{T}} \leq c(\gamma)\left\|h^{\prime \prime}\right\|_{1+1 / 2,0, S^{T}} .
\end{aligned}
$$

On using the above extensions, problem (4.20) takes the form

$$
\begin{aligned}
& \widetilde{w}_{t}-\mu \nabla^{2} \widetilde{w}-\nu \nabla \operatorname{div} \widetilde{w}=f^{\prime \prime} \\
& \text { in } \Omega \times(-\infty, T) \text {, } \\
& \Pi_{0} \mathbb{D}(\widetilde{w}) \bar{n}_{0}=g_{1}^{\prime \prime} \\
& \text { on } S \times(-\infty, T) \text {, } \\
& \bar{n}_{0} \mathbb{D}(\widetilde{w}) \bar{n}_{0}-\sigma \bar{n}_{0} \Delta_{S}(0) \int_{0}^{t} \widetilde{w}(\tau) d \tau \\
& =g_{2}^{\prime}+\sigma \int_{0}^{t} h^{\prime \prime}(\tau) d \tau \quad \text { on } S \times(-\infty, T),
\end{aligned}
$$

where $\widetilde{w}$ is zero for $t<0$ and $\widetilde{w}=w$ for $t \geq 0$.

In view of Theorem 4.3 and $(4.22),(4.23),(4.25),(4.26)$ there exists a solution of problem (4.27) such that $\widetilde{w} \in H_{\gamma}^{4,2}\left(S^{T}\right)$ and

$$
\|\widetilde{w}\|_{4, \gamma, \Omega^{T}} \leq c(\gamma)\left(X_{1}+X_{2}\right) .
$$

Now (4.19), (4.18), (4.28) and the equivalence of the norms of $H_{0}^{l, l / 2}\left(\Omega^{T}\right)$ and $H_{\gamma}^{l, l / 2}\left(\Omega^{T}\right)$ for $T<\infty$ imply

$$
\begin{aligned}
\|u\|_{4, \Omega^{T}} & \leq\|w\|_{4, \Omega^{T}}+\|v\|_{4, \Omega^{T}} \leq\|w\|_{4,0, \Omega^{T}}+c X_{2} \\
& \leq c(\gamma)\|w\|_{4, \gamma, \Omega^{T}}+c X_{2} \leq c(\gamma)\left(X_{1}+X_{2}\right) .
\end{aligned}
$$

Hence (4.29) implies (4.16). This concludes the proof.

To prove Theorem 4.1 we needed the following result:

Lemma 4.5 (see also [2], Section 3, Ch. 2, Theorem 21). Let $\phi_{0}, \ldots, \phi_{k}$, $\phi_{j} \in H^{l-2 j-1}\left(\mathbb{R}^{n}\right), l-2 k-1 \geq 0, l, k \in \mathbb{N} \cup\{0\}$, be given. Then there exists a function $u \in W_{2}^{l, l / 2}\left(\mathbb{R}^{n} \times \mathbb{R}_{+}\right)$such that

$$
\left.\frac{\partial^{j} u}{\partial t^{j}}\right|_{t=0}=\phi_{j}(x), \quad j=0, \ldots, k,
$$

and

$$
\|u\|_{l, \mathbb{R}^{n} \times \mathbb{R}_{+}} \leq c \sum_{j=0}^{k}\left\|\phi_{j}\right\|_{l-2 j-1, \mathbb{R}^{n}},
$$

where the constant $c$ does not depend on $\phi_{j}, j=0, \ldots, k$. 
P r o o f. Assume that $\phi_{j} \in C_{0}^{\infty}\left(\mathbb{R}^{n}\right)$. Define a Fourier transform of $u(x, t)$ with respect to the variables $x=\left(x_{1}, \ldots, x_{n}\right)$ by

$$
\widehat{u}(\xi, t)=\sum_{j=0}^{k} \frac{\Phi_{j}\left(\left(1+\xi^{2}\right) t\right)}{\left(1+\xi^{2}\right)^{j}} \widehat{\phi}_{j}(\xi), \quad \xi=\left(\xi_{1}, \ldots, \xi_{n}\right),
$$

where the $\Phi_{j} \in C_{0}^{\infty}\left(\mathbb{R}^{1}\right)$ satisfy the relations

$$
\left.\frac{d^{i} \Phi_{j}(s)}{d s^{i}}\right|_{s=0}=\delta_{i j}, \quad i, j=0, \ldots, k,
$$

and $\widehat{\phi}_{j}(\xi)$ is the Fourier transform of $\phi_{j}$.

We can take $\Phi_{j}(s)=\left(s^{j} / j !\right) \Phi_{0}(s), \Phi_{0} \in C_{0}^{\infty}\left(\mathbb{R}^{1}\right), \Phi_{0}(s)=1$ for small $s$.

Taking the Fourier transform of (4.32) with respect to $t$ gives

$$
\widehat{u}\left(\xi, \xi_{0}\right)=\sum_{j=0}^{k} \frac{\widehat{\Phi}_{j}\left(\xi_{0} /\left(1+\xi^{2}\right)\right)}{\left(1+\xi^{2}\right)^{j+1}} \widehat{\phi}_{j}(\xi)
$$

where $\widehat{\Phi}_{j}$ is the Fourier transform of $\Phi_{j}$.

Now we estimate the norm

$$
\begin{aligned}
\|u\|_{l, \mathbb{R}^{n} \times \mathbb{R}_{+}}^{2} & =\int_{\mathbb{R}^{n} \times \mathbb{R}}\left(1+|\xi|^{2}+\xi_{0}\right)^{l}\left|\sum_{j=0}^{k} \widehat{\Phi}_{j}\left(\frac{\xi_{0}}{1+\xi^{2}}\right) \frac{\widehat{\phi}_{j}(\xi)}{\left(1+\xi^{2}\right)^{j+1}}\right|^{2} d \xi d \xi_{0} \\
& \leq \sum_{j=0}^{k} \int_{\mathbb{R}^{n}} \frac{\left|\widehat{\phi}_{j}(\xi)\right|^{2}}{\left|1+\xi^{2}\right|^{2 j+2}} d \xi \int_{0}^{\infty}\left|\widehat{\Phi}_{j}\left(\frac{\xi_{0}}{1+\xi^{2}}\right)\right|^{2}\left(1+|\xi|^{2}+\xi_{0}\right)^{l} d \xi_{0} .
\end{aligned}
$$

Introducing a new variable in the inner integral,

$$
\eta=\frac{\xi_{0}}{1+\xi^{2}}
$$

we get

$$
\begin{aligned}
\|u\|_{l, \mathbb{R}^{n} \times \mathbb{R}_{+}}^{2} & =\sum_{j=0}^{k} \int_{\mathbb{R}^{n}}\left|\widehat{\phi}_{j}(\xi)\right|^{2}\left(1+|\xi|^{2}\right)^{l-2 j-1} d \xi \int_{0}^{\infty}\left|\widehat{\Phi}_{j}(\eta)\right|^{2}(1+\eta)^{l} d \eta \\
& \leq c \sum_{j=0}^{k} \int_{\mathbb{R}^{n}}\left|\widehat{\phi}_{j}(\xi)\right|^{2}\left(1+|\xi|^{2}\right)^{l-2 j-1} d \xi
\end{aligned}
$$

Hence (4.31) follows. This concludes the proof.

5. Existence of solutions to problem (1.11). First we consider the following problem with $\eta>0$ : 


$$
\begin{aligned}
& \eta u_{t}-\mu \nabla_{\xi}^{2} u-\nu \nabla_{\xi} \nabla_{\xi} \cdot u=F \quad \text { in } \Omega^{T}, \\
& \Pi_{0} \mathbb{D}_{\xi}(u) \bar{n}_{0}=G_{1} \quad \text { on } S^{T},
\end{aligned}
$$

Lemma 5.1. Let $0<\eta \in C^{\alpha}\left(\Omega^{T}\right) \cap L_{\infty}\left(0, T ; \Gamma_{1}^{2}(\Omega)\right), 1 / \eta \in L_{\infty}\left(\Omega^{T}\right), f \in$ $W_{2}^{2,1}\left(\Omega^{T}\right), G_{i} \in W_{2,1 / 4}^{2+1 / 2,1+1 / 4}\left(S^{T}\right), i=1,2, H \in W_{2}^{2-1 / 2,1-1 / 4}\left(S^{T}\right)$, and $S \in H^{4-1 / 2}$. Then there exists a solution of (5.1) such that $u \in W_{2}^{4,2}\left(\Omega^{T}\right)$ and

(5.2) $\quad\|u\|_{4, \Omega^{T}}$

$$
\begin{aligned}
\leq & \phi_{1}\left(|1 / \eta|_{\infty, \Omega^{T}}, \sup _{t}|\eta|_{2,1, \Omega}\right)\|u\|_{2, \Omega^{T}} \\
& +\phi_{2}\left(|1 / \eta|_{\infty, \Omega^{T}},|\eta|_{\infty, \Omega^{T}},|\eta|_{C^{\alpha}\left(\Omega^{T}\right)}\right) \\
& \times\left[\|F\|_{2, \Omega^{T}}+\|G\|_{2+1 / 2, S^{T}, 1 / 4}+\|H\|_{2-1 / 2, S^{T}}+|u(0)|_{3,0, \Omega}\right],
\end{aligned}
$$

where $\phi_{1}, \phi_{2}$ are increasing functions of their arguments and $G=\left(G_{1}, G_{2}\right)$.

P r o of. First we consider problem (5.1) with vanishing initial data. Introducing a partition of unity $\zeta^{(k, l)}(\xi, t)$ in $\Omega^{T}$ such that $\operatorname{supp} \zeta^{(k, l)}(\xi, t) \subset$ $\Omega_{k} \times\left(T_{l-1}, T_{l}\right)$ (see Section 2) and setting $u_{(k, l)}=u \zeta^{(k, l)}$ we write problem (5.1) locally in the form (see [13])

$$
\begin{gathered}
\eta\left(\xi_{k}, t_{l}\right) u_{(k, l) t}-\mu \nabla_{\xi}^{2} u_{(k, l)}-\nu \nabla_{\xi} \nabla_{\xi} \cdot u_{(k, l)} \\
=\left[\eta\left(\xi_{k}, t_{l}\right)-\eta(\xi, t)\right] u_{(k, l) t}+\eta u \zeta_{t}^{(k, l)}-\mu\left[\nabla_{\zeta}^{2}, \zeta^{(k, l)}\right] u \\
-\nu\left[\nabla_{\xi} \nabla_{\xi}, \zeta^{(k, l)}\right] u+F_{(k, l)} \equiv F_{(k, l)}^{\prime}+F_{(k, l)} \equiv \widetilde{F}_{(k, l)}, \\
\Pi_{0} \mathbb{D}_{\xi}\left(u_{(k, l)}\right) \bar{n}_{0}=\Pi_{0} \mathbb{D}_{\xi}\left(\zeta^{(k, l)}\right) \bar{n}_{0} u+G_{1(k, l)} \\
\equiv G_{1(k, l)}^{\prime}+G_{1(k, l)} \equiv \widetilde{G}_{1(k, l)}, \\
\bar{n}_{0} \mathbb{D}_{\xi}\left(u_{(k, l)}\right) \bar{n}_{0}-\sigma \bar{n}_{0} \Delta_{S}(0) \int_{0}^{t} u_{(k, l)}(\tau) d \tau \\
=\bar{n}_{0} \mathbb{D}_{\xi}\left(\zeta^{(k, l)}\right) \bar{n}_{0} u+G_{2(k, l)} \\
+\int_{0}^{t}\left(-\sigma \bar{n}_{0}\left[\Delta_{S}(0), \zeta^{(k, l)}\right] u+\bar{n}_{0} \mathbb{D}_{\xi}(u) \zeta_{, \tau}^{(k, l)} \bar{n}_{0}\right) d \tau \\
+\int_{0}^{t}\left(-G_{2} \xi_{, \tau}^{(k, l)}+H_{(k, l)}(\tau)\right) d \tau
\end{gathered}
$$




$$
\begin{aligned}
& \equiv G_{2(k, l)}^{\prime}+G_{2(k, l)}+\sigma \int_{0}^{t}\left(H_{(k, l)}^{\prime}(\tau)+H_{(k, l)}(\tau)\right) d \tau \\
& \equiv \widetilde{G}_{2(k, l)}+\sigma \int_{0}^{t} \widetilde{H}_{(k, l)}(\tau) d \tau \\
\left.u_{(k, l)}\right|_{t=0} & =0
\end{aligned}
$$

where we have used the notation $K_{(k, l)}=K \zeta^{(k, l)}, K \in\left\{F, G_{1}, G_{2}, H\right\}$.

Introducing a new variable $\tau=\eta_{k l}^{-1} t, \eta_{k l}=\eta\left(\xi_{k}, t_{l}\right)$, where $\xi_{k} \in \Omega_{k}$, $t_{l} \in\left(T_{l-1}, T_{l}\right)$, applying Theorem 4.4 and then going back to the variable $t$ we have

$$
\begin{aligned}
\left\|u_{(k, l)}\right\|_{4, \Omega^{T}} \leq & \phi\left(1 / \eta_{k l}, \eta_{k l}, T\right) \\
& \times\left(\left\|\widetilde{F}_{(k, l)}\right\|_{2, \Omega^{T}}+\left\|\widetilde{G}_{(k, l)}\right\|_{(2+1 / 2), S^{T}, 1 / 4}\right. \\
& \left.+\left\|\widetilde{H}_{(k, l)}\right\|_{1+1 / 2, S^{T}}+\left\|F_{(k, l)}(0)\right\|_{1, \Omega}\right),
\end{aligned}
$$

where $\phi$ is a positive increasing function of its arguments.

Now we estimate the particular terms on the right-hand side of (5.4). First we consider

$$
\begin{aligned}
& \left\|F_{(k, l)}^{\prime}\right\|_{2, \Omega^{T}} \\
& \leq c \lambda^{\alpha}|\eta|_{C^{\alpha}\left(\Omega^{T}\right)}\left\|u_{(k, l)}\right\|_{4, \Omega^{T}} \\
& \quad+c\left(\int_{0}^{T} \int_{\Omega}\left(\left|\nabla \eta \nabla u_{(k, l) t}\right|^{2}+\left|\nabla^{2} \eta u_{(k, l) t}\right|^{2}+\left|\eta_{t} u_{(k, l) t}\right|^{2}\right) d \xi d t\right)^{1 / 2} \\
& \quad+c\left(\|\nabla u\|_{2, \Omega_{k} \times\left(T_{l-1}, T_{l}\right)}+\|u\|_{2, \Omega_{k} \times\left(T_{l-1}, T_{l}\right)}\right),
\end{aligned}
$$

where the middle term is estimated by

$$
c \sup _{t}|\eta|_{2,1, \Omega}\left(\varepsilon\left\|u_{(k, l)}\right\|_{4, \Omega^{T}}+c(\varepsilon)\left\|u_{(k, l)}\right\|_{2, \Omega^{T}}\right), \quad \varepsilon \in(0,1) .
$$

Next, we consider

$$
\begin{aligned}
\left\|G_{(k, l)}^{\prime}\right\|_{2+1 / 2, S^{T}, 1 / 4} \leq & c\left(\left\|\bar{n}_{0} u\right\|_{2+1 / 2, S_{k} \times\left(T_{l-1}, T_{l}\right), 1 / 4}\right. \\
& \left.+\left\|\bar{n}_{0} \bar{n}_{0} u\right\|_{2+1 / 2, S_{k} \times\left(T_{l-1}, T_{l}\right), 1 / 4}\right),
\end{aligned}
$$

where $S_{k}=S \cap \bar{\Omega}_{k}$. To estimate the right-hand side of (5.6) it is sufficient to find a bound for

$$
\left\|\bar{n}_{0} u\right\|_{2+1 / 2, S_{k} \times\left(T_{l-1}, T_{l}\right)}+\left(\int_{T_{l-1}}^{T_{l}} \frac{|u|_{2,0, S_{k}}^{2}}{t^{1 / 4}} d t\right)^{1 / 2} \equiv I_{1}+I_{2} .
$$

To estimate $I_{1}$ and $I_{2}$ we consider only the highest order terms. First we estimate the expression 
$\left[\bar{n}_{0} u\right]_{2+1 / 2, S_{k} \times\left(T_{l-1}, T_{l}\right), x}$

$$
\begin{aligned}
\leq & \sum_{|\bar{\alpha}| \leq 2}\left(\int_{T_{l-1}}^{T_{l}} \int_{S_{k}} \int_{S_{k}} \frac{\left|D_{\xi, t}^{\bar{\alpha}} u-D_{\xi^{\prime}, t}^{\bar{\alpha}} u\right|^{2}\left|D_{\xi}^{2-\alpha} \bar{n}_{0}\right|^{2}}{\left|\xi-\xi^{\prime}\right|^{3}} d \xi d \xi^{\prime} d t\right)^{1 / 2} \\
& +\sum_{|\bar{\alpha}|=2}\left(\int_{T_{l-1}}^{T_{l}} \int_{S_{k}} \int_{S_{k}} \frac{\left|D_{\xi, t}^{\bar{\alpha}} u\right|^{2}\left|D_{\xi}^{2-\alpha} \bar{n}_{0}-D_{\xi^{\prime}}^{2-\alpha} \bar{n}_{0}\right|^{2}}{\left|\xi-\xi^{\prime}\right|^{3}} d \xi d \xi^{\prime} d t\right)^{1 / 2} \\
\leq & c\left(\int_{T_{l-1}}^{T_{l}} \int_{S_{k}} \int_{S_{k}} \frac{\left|u(\xi)-u\left(\xi^{\prime}\right)\right|^{2}}{\left|\xi-\xi^{\prime}\right|^{3}}\left|D_{\xi}^{2} \bar{n}_{0}(\xi)\right|^{2} d \xi d \xi^{\prime} d t\right)^{1 / 2} \\
& +\phi\left(\left\|S_{k-1}\right\|_{4-1 / 2}\right) \\
& \times\left(\int_{T_{l-1}}^{T_{l}}\left(\left[D_{\xi, t}^{2}\right]_{1 / 2, S_{k}}^{2}+\left[D_{\xi} u\right]_{1 / 2, S_{k}}^{2}+\left|D_{\xi, t}^{2} u\right|_{0, S_{k}}^{2}+\left|D_{\xi} u\right|_{0, S_{k}}^{2}\right) d t\right)^{1 / 2} \\
& +c\left(\int_{T_{l-1}}^{T_{l}}|u|_{\infty, S_{k}}^{2} d t\right)^{1 / 2}\left[D_{\xi}^{2} \bar{n}_{0}\right]_{1 / 2, S_{k}},
\end{aligned}
$$

where $\phi$ is a positive increasing function, $D_{\xi, t}^{2}=\sum_{|\bar{\alpha}|=2} D_{\xi, t}^{\bar{\alpha}}, D_{\xi}^{2}=$ $\sum_{|\alpha|=2} D_{\xi}^{\alpha}, \bar{\alpha}=\left(\alpha_{0}, \alpha\right)$.

Using the interpolation inequalities (see [1], Secs. 10, 18)

$$
\begin{gathered}
{\left[D_{\xi}^{2} u\right]_{1 / 2, S_{k}}+\left[D_{\xi} u\right]_{1 / 2, S_{k}}+\|u\|_{2, S_{k}} \leq \varepsilon\|u\|_{4, \Omega_{k}}+c(\varepsilon)\|u\|_{0, \Omega_{k}},} \\
{\left[\partial_{t} u\right]_{1 / 2, S_{k}}+\left|\partial_{t} u\right|_{0, S_{k}} \leq \varepsilon\left\|u_{t}\right\|_{2, \Omega_{k}}+c(\varepsilon)\|u\|_{0, \Omega_{k}},}
\end{gathered}
$$

we obtain

$$
\begin{aligned}
& {\left[\bar{n}_{0} u\right]_{2+1 / 2, S_{k} \times\left(T_{l-1}, T_{l}\right), x} } \\
\leq & \varepsilon\|u\|_{4, \Omega_{k} \times\left(T_{l-1}, T_{l}\right)}+c\left(\varepsilon,\left\|S_{k}\right\|_{4-1 / 2}\right)\|u\|_{2, \Omega_{k} \times\left(T_{l-1}, T_{l}\right)}, \quad \varepsilon \in(0,1) .
\end{aligned}
$$

Now we examine

$$
\begin{aligned}
& \leq \phi\left(\left\|S_{k}\right\|_{4-1 / 2}\right)\left(\int_{T_{l-1}}^{T_{l}} \int_{T_{l-1}}^{T_{l}} \frac{\left\|u(t)-u\left(t^{\prime}\right)\right\|_{2, S_{k}}^{2}+\left\|\partial_{t} u-\partial_{t^{\prime}} u\right\|_{0, S_{k}}^{2}}{\left|t-t^{\prime}\right|^{3 / 2}} d t d t^{\prime}\right)^{1 / 2} \\
& \leq \varepsilon\|u\|_{4, \Omega_{k} \times\left(T_{l-1}, T_{l}\right)}+c(\varepsilon)\|u\|_{2, \Omega_{k} \times\left(T_{l-1}, T_{l}\right)},
\end{aligned}
$$

where the last inequality follows from interpolation inequalities (see [1], Sec. 18) and $c(\varepsilon)$ depends also on the length of the interval $\left[T_{l-1}, T_{l}\right]$, which is fixed. 
Finally, the second term in (5.7) is bounded by

$$
\begin{aligned}
& \left(\int_{T_{l-1}}^{T_{l}} \frac{\varepsilon\left|D_{\xi, t}^{3} u\right|_{2, \Omega_{k}}^{2}+c(\varepsilon)\left|D_{\xi, t}^{2} u\right|_{2, \Omega_{k}}^{2}}{t^{1 / 2}} d t\right)^{1 / 2} \\
& \quad \leq \varepsilon\left(\|u\|_{4, \Omega_{k} \times\left(T_{l-1}, T_{l}\right)}+\sup _{t \in\left(T_{l-1}, T_{l}\right)}|u|_{3,0, \Omega_{k}}\right)+c\|u\|_{2, \Omega_{k} \times\left(T_{l-1}, T_{l}\right)} .
\end{aligned}
$$

Summarizing the above considerations we obtain

$$
\begin{aligned}
& \left\|u_{(k, l)}\right\|_{4, \Omega^{T}} \\
& \leq c \lambda^{\alpha}|\eta|_{C^{\alpha}\left(\Omega^{T}\right)}\left\|u_{(k, l)}\right\|_{4, \Omega^{T}} \\
& \quad+\varepsilon\left(\left\|u_{(k, l)}\right\|_{4, \Omega^{T}}+\|u\|_{4, \Omega_{k} \times\left(T_{l-1}, T_{l}\right)}+\sup _{t \in\left(T_{l-1}, T_{l}\right)}|u|_{3,0, \Omega_{k}}\right) \\
& \quad+\widetilde{\phi}_{1}\left(|1 / \eta|_{\infty, \Omega^{T}}, \sup _{t}|\eta|_{2,1, \Omega}\right)\left(\left\|u_{(k, l)}\right\|_{2, \Omega^{T}}+\|u\|_{2, \Omega_{k} \times\left(T_{l-1}, T_{l}\right)}\right) \\
& \quad+\widetilde{\phi}_{2}\left(|1 / \eta|_{\infty, \Omega^{T}},|\eta|_{\infty, \Omega^{T}}\right) \\
& \quad \times\left(\left\|F_{(k, l)}\right\|_{2, \Omega^{T}}+\left\|G_{(k, l)}\right\|_{2+1 / 2, S^{T}, 1 / 4}+\left\|H_{(k, l)}\right\|_{2-1 / 2, S^{T}}\right),
\end{aligned}
$$

where $\widetilde{\phi}_{1}, \widetilde{\phi}_{2}$ are positive increasing functions. Summing (5.10) over all subdomains of the partition of unity and using the fact that $\lambda$ and $\varepsilon$ are sufficiently small we obtain (5.2) for vanishing initial data.

To obtain (5.2) for nonvanishing initial data we write problem (5.1) in the form of two problems

$$
\begin{aligned}
& \omega_{t}-\operatorname{div}_{\xi} \mathbb{D}_{\xi}(\omega)=F, \\
& \Pi_{0} \mathbb{D}_{\xi}(\omega) \bar{n}_{0}=0, \\
& \bar{n}_{0} \mathbb{D}_{\xi}(\omega) \bar{n}_{0}-\sigma \bar{n}_{0} \Delta_{S}(0) \int_{0}^{t} \omega(\tau) d \tau=0, \\
& \left.\omega\right|_{t=0}=u_{0},
\end{aligned}
$$

and

$$
\begin{aligned}
& \eta v_{t}-\operatorname{div}_{\xi} \mathbb{D}_{\xi}(v)=(1-\eta) \omega_{t}, \\
& \Pi_{0} \mathbb{D}_{\xi}(v) \bar{n}_{0}=G_{1}, \\
& \bar{n}_{0} \mathbb{D}_{\xi}(v) \bar{n}_{0}-\sigma \bar{n}_{0} \Delta_{S}(0) \int_{0}^{t} v(\tau) d \tau=G_{2}+\sigma \int_{0}^{t} H(\tau) d \tau, \\
& \left.v\right|_{t=0}=0,
\end{aligned}
$$

where $u=v+\omega$.

Applying Theorem 4.4 to the first problem and estimate (5.2) for solutions of the second problem, which has just been shown above, we obtain (5.2) for solutions of (5.1). 
We prove existence of solutions to (5.1) by the method of successive approximations. We put $u_{m+1}$ into the left-hand sides of (5.3) and $u_{m}$ into the right-hand sides. In view of estimate (5.10) the sequence converges for sufficiently small $\lambda$ and $\varepsilon$. This concludes the proof.

Now we examine the problem

$$
\begin{aligned}
& \eta u_{t}-\mu \nabla_{\omega}^{2} u-\nu \nabla_{\omega} \nabla_{\omega} \cdot u=F, \\
& \Pi_{0} \mathbb{D}_{\omega}(u) \bar{n}_{0}=G_{1}, \\
& \bar{n}_{0} \mathbb{D}_{\omega}(u) \bar{n}_{0}-\sigma \bar{n}_{0} \Delta_{S_{t}}(t) \int_{0}^{t} u(\tau) d \tau=G_{2}+\sigma \int_{0}^{t} H(\tau) d \tau, \\
& \left.u\right|_{t=0}=u_{0} .
\end{aligned}
$$

LEMMA 5.2. Let the assumptions of Lemma 5.1 be satisfied. Let $\omega \in$ $W_{2}^{4,2}\left(\Omega^{T}\right)$. There exists a function $\phi_{3}$ and $T$ such that if

$$
\begin{aligned}
T^{1 / 2} \phi_{3}\left(T ^ { 1 / 4 } \left(\|\omega\|_{4, \Omega^{T}}\right.\right. & \left.\left.+\sup _{t}|\omega|_{3,0, \Omega}\right), T\right) \\
& \times \phi_{2}\left(|1 / \eta|_{\infty, \Omega^{T}},|\eta|_{\infty, \Omega^{T}},|\eta|_{C^{\alpha}\left(\Omega^{T}\right)}\right) \leq \delta
\end{aligned}
$$

then there exists a solution to problem (5.11) for $\delta$ sufficiently small such that $u \in W_{2}^{4,2}\left(\Omega^{T}\right)$ and

$$
\begin{aligned}
\|u\|_{4, \Omega^{T}} \leq & c \phi_{2}\left(\|F\|_{2, \Omega^{T}}+\|G\|_{(2+1 / 2), S^{T}, 1 / 4}\right. \\
& \left.+\|H\|_{2-1 / 2, S^{T}}+|u(0)|_{3,0, \Omega}\right)+c \phi_{1}\|u\|_{3, \Omega^{T}} .
\end{aligned}
$$

Proof. We write problem (5.11) in the form

$$
\begin{gathered}
\eta u_{t}-\mu \nabla_{\xi}^{2} u-\nu \nabla_{\xi} \nabla_{\xi} \cdot u \\
=-\mu\left(\nabla_{\xi}^{2}-\nabla_{\omega}^{2}\right) u-\nu\left(\nabla_{\xi} \nabla_{\xi} \cdot-\nabla_{\omega} \nabla_{\omega} \cdot\right) u+F \equiv \widetilde{F}+F, \\
\Pi_{0} \mathbb{D}_{\xi}(u) \bar{n}_{0}=\Pi_{0} \mathbb{D}_{\xi}(u) \bar{n}_{0}-\Pi_{0} \Pi \mathbb{D}_{\omega}(u) \bar{n}_{0}+G_{1} \equiv \widetilde{G}_{1}+G_{1} \\
\bar{n}_{0} \mathbb{D}_{\xi}(u) \bar{n}_{0}-\sigma \bar{n}_{0} \Delta_{S}(0) \int_{0}^{t} u(\tau) d \tau \\
=\bar{n}_{0} \mathbb{D}_{\xi}(u)\left(\bar{n}_{0}-\bar{n}\right)+\bar{n}_{0}\left(\mathbb{D}_{\xi}(u)-\mathbb{D}_{\omega}(u)\right) \bar{n} \\
\quad-\sigma \bar{n}_{0}\left(\Delta_{S}(0)-\Delta_{S_{t}}(t)\right) \int_{0}^{t} u(\tau) d \tau+G_{2}+\sigma \int_{0}^{t} H(\tau) d \tau \\
\equiv \widetilde{G}_{t=0}+G_{2}+\sigma \int_{0}^{t}(\widetilde{H}(\tau)+H(\tau)) d \tau \\
u
\end{gathered}
$$


Using Lemma 5.1 we have the following estimate for a solution of (5.14):

$$
\begin{aligned}
\|u\|_{4, \Omega^{T}} \leq & \phi_{1}\left(|1 / \eta|_{\infty, \Omega^{T}}, \sup _{t}|\eta|_{2,1, \Omega}\right)\|u\|_{2, \Omega^{T}} \\
& +\phi_{2}\left(|1 / \eta|_{\infty, \Omega^{T}},|\eta|_{\infty, \Omega^{T}},|\eta|_{C^{\alpha}\left(\Omega^{T}\right)}\right)\left[\|\widetilde{F}+F\|_{2, \Omega^{T}}\right. \\
& \left.+\|\widetilde{G}+G\|_{2+1 / 2, S^{T}, 1 / 4}+\|\widetilde{H}+H\|_{2-1 / 2, S^{T}}+|u|_{3,0, \Omega}\right] .
\end{aligned}
$$

Now we have to estimate the particular terms on the right-hand side of (5.15). The functions $\widetilde{F}, \widetilde{G}$ and $\widetilde{H}$ have the following qualitative forms:

$$
\begin{aligned}
& \widetilde{F}=f_{1} \int_{0}^{t} \omega_{\xi \xi} d \tau u_{\xi}+f_{2} \int_{0}^{t} \omega_{\xi} d \tau u_{\xi \xi}, \\
& \widetilde{G}=\left.f_{3}\left(\int_{0}^{t} \omega_{\xi} d \tau u_{\xi}\right)\right|_{S}, \\
& \widetilde{H}=\left.f_{4}\left(\int_{0}^{t} \omega_{\xi \xi} d \tau u_{\xi}+\int_{0}^{t} u_{\xi} d \tau u_{\xi \xi}\right)\right|_{S},
\end{aligned}
$$

where $f_{i}, i=1, \ldots, 4$, depend on $\delta+\int \omega_{\xi} d \tau$, where $\delta$ is the unit matrix, and $f_{3}, f_{4}$ depend additionally on $\nabla \widetilde{\phi}$, where $\widetilde{\phi}(\xi)=0$ describes $S$ locally.

In view the Hölder inequality and imbedding theorems we have

$$
\begin{aligned}
\|\widetilde{F}\|_{2, \Omega^{T}}+ & \|\widetilde{G}\|_{2+1 / 2, S^{T}, 1 / 4}+\|\widetilde{H}\|_{2-1 / 2, S^{T}} \\
\leq & c T^{1 / 4}\left(\|\omega\|_{4, \Omega^{T}}+\sup _{t}|\omega|_{3,0, \Omega}\right) \\
& \times \psi_{1}\left(T^{1 / 2}\|\omega\|_{4, \Omega^{T}}, T\right) T^{1 / 4}\left(\|u\|_{4, \Omega^{T}}+\sup _{t}|u|_{3,0, \Omega}\right) \\
\equiv & c \phi_{3}\left(T^{1 / 4}\left(\|\omega\|_{4, \Omega^{T}}+\sup _{t}|\omega|_{3,0, \Omega}\right), T\right) \\
& \times T^{1 / 2}\left(\|u\|_{4, \Omega^{T}}+\sup _{t}|u|_{3,0, \Omega}\right),
\end{aligned}
$$

where $\psi_{1}$ and $\phi_{3}$ are increasing functions of their arguments and $c$ does not depend on $T$.

From (5.15), (5.17) we obtain (5.13) for sufficiently small $\delta$.

Existence can be proved by the method of successive approximations. This concludes the proof.

6. Existence of solutions to problem (1.1). First we consider the continuity equation $(1.8)_{2}$ with the initial condition $(1.8)_{5}$. By the method of characteristics we have

$$
\eta(\xi, t)=\varrho_{0}(\xi) \exp \left[-\int_{0}^{t} \nabla_{u} \cdot u(\xi, \tau) d \tau\right]
$$


Lemma 6.1. Assume that $u \in W_{2}^{4,2}\left(\Omega^{T}\right) \cap L_{\infty}\left(0, T ; \Gamma_{0}^{3}(\Omega)\right), \varrho_{0} \in H^{3}(\Omega)$, and $T<\infty$. Then the solution (6.1) of problem $(1.1)_{2,3}$ is such that $\eta \in C_{0}\left([0, T] ; H^{3}(\Omega)\right), \eta_{t} \in C_{0}\left([0, T] ; H^{2}(\Omega)\right) \cap L_{2}\left(0, T ; H^{3}(\Omega)\right), \eta_{t t} \in$ $L_{2}\left(0, T ; H^{1}(\Omega)\right)$ and the following estimates hold:

(6.2) $\sup _{t}\|\eta\|_{3, \Omega} \leq c\left\|\varrho_{0}\right\|_{3, \Omega} \psi_{1}\left(T^{1 / 2}\|u\|_{4, \Omega^{T}}\right)\left(T^{1 / 2}\|u\|_{4, \Omega^{T}}+1\right)$,

(6.3) $\sup _{t}\left\|\eta_{t}\right\|_{2, \Omega} \leq c\left\|\varrho_{0}\right\|_{3, \Omega} \psi_{2}\left(T^{1 / 2}\|u\|_{4, \Omega^{T}}\right)\left(\|u\|_{4, \Omega^{T}}+\|u(0)\|_{3, \Omega}\right)$,

(6.4) $\left\|\eta_{t}\right\|_{L_{2}\left(0, T ; H^{3}(\Omega)\right)} \leq c\left\|\varrho_{0}\right\|_{3, \Omega} \psi_{3}\left(T^{1 / 2}\|u\|_{4, \Omega^{T}}\right)\|u\|_{4, \Omega^{T}}$, $\left\|\eta_{t t}\right\|_{L_{2}\left(0, T ; H^{1}(\Omega)\right)} \leq c\left\|\varrho_{0}\right\|_{3, \Omega} \psi_{4}\left(T^{1 / 2}\|u\|_{4, \Omega^{T}}\right)\|u\|_{4, \Omega^{T}}$, $\sup _{t}\left\|\eta_{t t}\right\|_{0, \Omega}$

$$
\leq c\left\|\varrho_{0}\right\|_{3, \Omega} \psi_{4}^{\prime}\left(T^{1 / 2}\|u\|_{4, \Omega^{T}},|u(0)|_{3,0, \Omega}\right)\left(\|u\|_{4, \Omega^{T}}+|u(0)|_{3,0, \Omega}\right),
$$

(6.6) $|1 / \eta|_{\infty, \Omega^{T}}+|\eta|_{\infty, \Omega^{T}} \leq\left(\left|1 / \varrho_{0}\right|_{\infty, \Omega}+\left|\varrho_{0}\right|_{\infty, \Omega}\right) \exp \left(T^{1 / 2}\|u\|_{4, \Omega^{T}}\right)$,

(6.7) $\quad\|\eta\|_{C^{\alpha}\left(\Omega^{T}\right)} \leq\left\|\varrho_{0}\right\|_{C^{\alpha}(\Omega)} \psi_{5}\left(T^{1 / 2}\|u\|_{4, \Omega^{T}}\right)$

$$
\times\left(\psi_{6}\left(T^{1 / 2}\|u\|_{4, \Omega^{T}}\right)+T^{1-\alpha}\left(\|u\|_{4, \Omega^{T}}+\|u(0)\|_{3, \Omega}\right)\right),
$$

where $\psi_{i}, i=1, \ldots, 6$, and $\psi_{4}^{\prime}$ are positive increasing functions.

Proof. First we show (6.2). We calculate

(6.8) $\quad\|\eta\|_{3, \Omega}$

$$
\begin{aligned}
& \leq c\left\|\varrho_{0}\right\|_{3, \Omega}\left\|\exp \left[-\int_{0}^{t} \nabla_{u} \cdot u d \tau\right]\right\|_{3, \Omega} \\
& \leq c\left\|\varrho_{0}\right\|_{3, \Omega} \exp \left|\int_{0}^{t} \nabla_{u} \cdot u d \tau\right|_{\infty, \Omega} \\
& \quad \times\left(1+\left\|\int_{0}^{t} \nabla_{u} \cdot u d \tau\right\|_{1, \Omega}^{3}\right. \\
& \left.\quad+\left\|\int_{0}^{t} \nabla_{u} \cdot u d \tau\right\|_{1, \Omega}\left\|\int_{0}^{t} \nabla_{u} \cdot u d \tau\right\|_{2, \Omega}+\left\|\int_{0}^{t} \nabla_{u} \cdot u d \tau\right\|_{3, \Omega}\right),
\end{aligned}
$$

and we have the estimates

$$
\begin{aligned}
\left|\int_{0}^{t} \nabla_{u} \cdot u d \tau\right|_{\infty, \Omega} & \leq c\left\|\int_{0}^{t} \nabla_{u} \cdot u d \tau\right\|_{2, \Omega} \leq c\left\|\int_{0}^{t} \nabla_{u} \cdot u d \tau\right\|_{3, \Omega} \\
& \leq \widetilde{\psi}_{1}\left(T^{1 / 2}\|u\|_{4, \Omega^{T}}\right) T^{1 / 2}\|u\|_{4, \Omega^{T}}
\end{aligned}
$$

where $\widetilde{\psi}_{1}$ is an increasing positive function. Using (6.9) in (6.8) implies (6.2). 
From (6.1) we have

$$
\eta_{t}=\varrho_{0} \exp \left[-\int_{0}^{t} \nabla_{u} \cdot u d \tau\right]\left(-\nabla_{u} \cdot u\right)
$$

so

$$
\left\|\eta_{t}\right\|_{2, \Omega} \leq c\left\|\varrho_{0}\right\|_{3, \Omega}\left\|\exp \left[-\int_{0}^{t} \nabla_{u} \cdot u d \tau\right]\right\|_{3, \Omega}\left\|\nabla_{u} \cdot u\right\|_{2, \Omega}
$$

In view of the Hölder inequality and imbedding theorems we have

$$
\begin{aligned}
\left\|\nabla_{u} \cdot u\right\|_{2, \Omega} & \leq \widetilde{\psi}_{2}\left(T^{1 / 2}\|u\|_{4, \Omega^{T}}\right)\|u\|_{3, \Omega} \\
& \leq \widetilde{\psi}_{2}\left(T^{1 / 2}\|u\|_{4, \Omega^{T}}\right)\left(\|u\|_{4, \Omega^{T}}+\|u(0)\|_{3, \Omega}\right),
\end{aligned}
$$

where the last inequality follows from Theorem 2 of [8] and $\widetilde{\psi}_{2}$ is a positive increasing function.

Using (6.8), (6.9) and (6.12) in (6.11) implies (6.3).

From (6.10) we have

$$
\left\|\eta_{t}\right\|_{3, \Omega} \leq c\left\|\varrho_{0}\right\|_{3, \Omega}\left\|\exp \left[-\int_{0}^{t} \nabla_{u} \cdot u d \tau\right]\right\|_{3, \Omega}\left\|\nabla_{u} \cdot u\right\|_{3, \Omega}
$$

Hence (6.4) holds.

From (6.10) we obtain

$$
\eta_{t t}=\varrho_{0} \exp \left[-\int_{0}^{t} \nabla_{u} \cdot u d \tau\right]\left[\left(\nabla_{u} \cdot u\right)^{2}-\nabla_{u} \cdot u_{t}+\widetilde{\psi}_{3}\left(\int_{0}^{t} u_{\xi} d \tau\right)\left(\nabla_{\xi} u\right)^{2}\right] .
$$

Therefore, (6.5) is valid. Similarly we show (6.6) and (6.7). This concludes the proof.

Finally, we prove the main result of the paper.

THEOREM 6.2. Assume that $v_{0}, \varrho_{0} \in H^{3}(\Omega), 1 / \varrho_{0} \in L_{\infty}(\Omega), S \in H^{1-1 / 2}$, and $f \in W_{2}^{2,1}\left(\Omega^{T}\right)$. Let $G$ be the function from (6.18) below and suppose $A>G(\gamma, 0,0)$, where $\gamma$ is defined in $(6.17)_{2}$. Let $|v(0)|_{3,0, \Omega}<A$. Let $\delta$ be sufficiently small. Let $T_{*}$ be so small that

$$
\begin{gathered}
T_{*}^{1 / 2} \phi_{3}\left(T_{*} A, T_{*}\right) \phi_{2}(A, A, A) \leq \delta \quad(\text { see }(5.12)), \\
0<c_{1}\left(1-A T_{*}\right)^{3} \leq \operatorname{det}\{\partial x / \partial \xi\} \leq c_{2}\left(1+A T_{*}\right)^{3},
\end{gathered}
$$

where $x=\xi+\int_{0}^{t} \widetilde{v}_{0}(\xi, \tau) d \tau, t \leq T_{*}, G\left(\gamma, T_{*}^{1 / 2} A, T_{*}\right)<A$ and $\widetilde{v}$ is defined below. Moreover, let compatibility conditions be satisfied (see proof below). Then there exists $T_{* *}, 0<T_{* *} \leq T_{*}$, such that for $T \leq T_{* *}$ there exists a unique solution to problem (1.1) such that $u \in W_{2}^{4,2}\left(\Omega^{T}\right)$, $\eta \in C\left([0, T] ; \Gamma_{0}^{3}(\Omega)\right), \eta_{t} \in L_{2}\left(0, T ; H^{3}(\Omega)\right), \eta_{t t} \in L_{2}\left(0, T ; H^{1}(\Omega)\right)$ and

$$
\|u\|_{4, \Omega^{T}} \leq A,
$$




$$
\begin{gathered}
\sup _{t}\|\eta\|_{3, \Omega}+\sup _{t}\left\|\eta_{t}\right\|_{2, \Omega}+\left\|\eta_{t}\right\|_{L_{2}\left(0, T, H^{3}(\Omega)\right)}+ \\
\quad\left\|\eta_{t t}\right\|_{L_{2}\left(0, T ; H^{1}(\Omega)\right)} \\
\leq \Phi_{1}\left(T, T^{a} A\right)\left\|\varrho_{0}\right\|_{3, \Omega}, \\
|1 / \eta|_{\infty, \Omega^{t}} \leq\left|1 / \varrho_{0}\right|_{\infty, \Omega} \Phi_{2}\left(T^{1 / 2} A\right)
\end{gathered}
$$

where $\Phi_{1}, \Phi_{2}$ are some increasing positive functions.

Proof. To prove existence of solutions to problem (1.1) we use the following method of successive approximations:

$$
\begin{aligned}
& \eta_{m} \partial_{t} u_{m+1}-\mu \nabla_{u_{m}}^{2} u_{m+1}-\nu \nabla_{u_{m}} \nabla_{u_{m}} \cdot u_{m+1} \\
& =-\nabla_{u_{m}} q\left(\eta_{m}\right)+\eta_{m} g, \\
& \Pi \mathbb{D}_{u_{m}}\left(u_{m+1}\right) \bar{n}\left(u_{m}\right)=0 \\
& \bar{n}_{0} \mathbb{D}_{u_{m}}\left(u_{m+1}\right) \bar{n}\left(u_{m}\right)-\sigma \bar{n}_{0} \Delta_{m}(t) \int_{0}^{t} u_{m+1}(\tau) d \tau \\
& =\bar{n}_{0} \cdot \bar{n}\left(u_{m}\right)\left(q\left(\eta_{m}\right)-p_{0}\right)+\sigma \bar{n}_{0} \Delta_{m}(t) \xi, \\
& \left.u_{m+1}\right|_{t=0}=v_{0},
\end{aligned}
$$

and

$$
\begin{aligned}
& \partial_{t} \eta_{m}+\eta_{m} \nabla_{u_{m}} \cdot u_{m}=0, \\
& \left.\eta_{m}\right|_{t=0}=\varrho_{0},
\end{aligned}
$$

where $m=0,1, \ldots$ and $u_{0}=\widetilde{v}_{0}$. Now we define $\widetilde{v}_{0}$. Let us introduce the functions $\phi^{i}=\left.\partial_{t}^{i} u\right|_{t=0}, i=0,1$, which are calculated from (1.8) $)_{1}$. The functions $\phi^{i}$ satisfy the following compatibility conditions:

$$
\left.\partial_{t}^{i}\left(\mathbb{T}_{u}(u, q) \bar{n}(\xi, t)-\sigma \Delta_{S_{t}} x(\xi, t)+p_{0} \bar{n}(\xi, t)\right)\right|_{t=0}=0, \quad i=0,1,
$$

where $\left.\partial_{t}^{i} u\right|_{t=0}$ and $\left.\partial_{t}^{i} \eta\right|_{t=0}, i=0,1$, have to be calculated from (1.8) $)_{1,4}$ and $(1.8)_{2,5}$, respectively. Next, we extend $\phi^{i}$ to functions $\widetilde{\phi}^{i}$ on $\mathbb{R}^{3}$, and define $\widetilde{v}$ to be the solution of the Cauchy problem

$$
\left(\partial_{t}-\Delta\right)^{2} \widetilde{v}=0,\left.\quad \partial_{t}^{i} \widetilde{v}\right|_{t=0}=\widetilde{\phi}^{i}, \quad i=0,1 .
$$

Finally, $\widetilde{v}_{0}=\left.\widetilde{v}\right|_{\Omega}$. First we obtain a uniform estimate. Applying Lemma 5.1 to $(6.13)$ yields

$$
\begin{aligned}
&\left\|u_{m+1}\right\|_{4, \Omega^{T}} \\
& \leq c \phi_{1}\left(\left|1 / \eta_{m}\right|_{\infty, \Omega^{T}}, \sup _{t}\left|\eta_{m}\right|_{2,1, \Omega}\right)\left\|u_{m+1}\right\|_{2, \Omega^{T}} \\
&+\varphi_{2}\left(\left|1 / \eta_{m}\right|_{\infty, \Omega^{T}},\left|\eta_{m}\right|_{\infty, \Omega^{T}},\left|\eta_{m}\right|_{C^{\alpha}\left(\Omega^{T}\right)}\right) \\
& \times\left[\left\|-\nabla_{u_{m}} q\left(\eta_{m}\right)+\eta_{m} g\right\|_{2, \Omega^{T}}\right. \\
&+\left\|\bar{n}_{0} \cdot \bar{n}\left(u_{m}\right)\left(q\left(\eta_{m}\right)-p_{0}\right)+\sigma \bar{n}_{0} \Delta_{m}(t) \xi\right\|_{2+1 / 2, S^{T}, 1 / 4} \\
&\left.+\left|u_{m+1}(0)\right|_{3,0, \Omega}\right] .
\end{aligned}
$$


Now we estimate the particular terms on the right-hand side of (6.15). First we consider

$$
\begin{aligned}
\|- & \nabla_{u_{m}} q\left(\eta_{m}\right)+\eta_{m} g \|_{2, \Omega^{T}} \\
\leq & \left\|\xi_{x}\left(u_{m}\right) q^{\prime}\left(\eta_{m}\right) \nabla \eta_{m}\right\|_{2, \Omega^{T}}+\left\|\eta_{m} g\right\|_{2, \Omega^{T}} \\
\leq & {\left[T^{1 / 2} \alpha_{1}\left(T^{1 / 2}\left\|u_{m}\right\|_{4, \Omega^{T}}, \sup _{t}\left\|\eta_{m}\right\|_{2, \Omega^{T}}\right)\left(1+\sup _{t}\left\|u_{m}\right\|_{3, \Omega}\right)+\|g\|_{2, \Omega^{T}}\right] } \\
& \left.\times \sup _{t}\left\|\eta_{m}\right\|_{3, \Omega}+\sup _{t}\left\|\eta_{m t}\right\|_{1, \Omega}\right),
\end{aligned}
$$

where $q^{\prime}(\eta)=d q / d \eta$ and $\alpha_{1}$ is an increasing positive function of its arguments.

Next, we have

$$
\begin{aligned}
\| \bar{n}_{0} \cdot \bar{n}\left(u_{m}\right)( & \left.q\left(\eta_{m}\right)-p_{0}\right) \|_{2+1 / 2, S^{T}} \\
\leq & \alpha_{2}\left(T^{1 / 2}\left\|u_{m}\right\|_{4, \Omega^{T}}, \sup _{t}\left\|\eta_{m}\right\|_{2, \Omega}, \sup _{t}\left\|\eta_{m t}\right\|_{1, \Omega^{T}}, T\right) \\
& \times\left[T^{1 / 4}\left\|u_{m}\right\|_{4, \Omega^{T}}+\int_{0}^{T}\left\|\eta_{m}\right\|_{3, \Omega}^{2} d \tau+\int_{0}^{T}\left\|\eta_{m t}\right\|_{1, \Omega}^{2} d \tau\right. \\
& +\left(\int _ { 0 } ^ { T } \int _ { 0 } ^ { T } \left(\frac{\left\|\eta_{m}(t)-\eta_{m}\left(t^{\prime}\right)\right\|_{2, S}^{2}}{\left|t-t^{\prime}\right|^{3 / 2}}\right.\right. \\
& \left.\left.\left.+\frac{\left\|\eta_{m}(t)-\eta_{m}\left(t^{\prime}\right)\right\|_{2, \Omega}^{2}+\left\|\eta_{m t}(t)-\eta_{m t^{\prime}}\left(t^{\prime}\right)\right\|_{0, S}^{2}}{\left|t-t^{\prime}\right|^{3 / 2}}\right) d t d t^{\prime}\right)^{1 / 2}\right],
\end{aligned}
$$

where

$$
\begin{gathered}
\left(\int_{0}^{T}\left\|\eta_{m}\right\|_{3, \Omega}^{2} d \tau\right)^{1 / 2} \leq\left\|\varrho_{0}\right\|_{3, \Omega} T^{1 / 2} \chi_{1}\left(T^{1 / 2}\left\|u_{m}\right\|_{4, \Omega^{T}}\right) \\
\left(\int_{0}^{T}\left\|\eta_{m t}\right\|_{1, \Omega}^{2} d \tau\right)^{1 / 2} \\
\leq\left\|\varrho_{0}\right\|_{2, \Omega} T^{1 / 2} \chi_{2}\left(T^{1 / 2}\left\|u_{m}\right\|_{4, \Omega^{T}}, T\right)\left(\left\|u_{m}\right\|_{3, \Omega^{T}}+\|u(0)\|_{2, \Omega}\right), \\
\int_{0}^{T} \int_{0}^{T}\left(\frac{\left\|\eta_{m}(t)-\eta_{m}\left(t^{\prime}\right)\right\|_{2, S}^{2}}{\left|t-t^{\prime}\right|^{3 / 2}}\right. \\
\left.\quad+\frac{\left\|\eta_{m}(t)-\eta_{m}\left(t^{\prime}\right)\right\|_{2, \Omega}^{2}+\left\|\eta_{m t}(t)-\eta_{m t^{\prime}}\left(t^{\prime}\right)\right\|_{0, S}^{2}}{\left|t-t^{\prime}\right| 3 / 2}\right) d t d t^{\prime} \\
\leq\left\|\varrho_{0}\right\|_{3, \Omega} \chi_{3}\left(T^{1 / 2}\left\|u_{m}\right\|_{4, \Omega^{T}}, T\right) T^{a}\left\|u_{m}\right\|_{4, \Omega^{T}},
\end{gathered}
$$

where $\chi_{i}, i=1,2,3$, are positive increasing functions, $a>0$. 
Next, we have

$$
\begin{aligned}
& \left\|\bar{n}_{0} \cdot \bar{n}\left(u_{m}\right)\left(q\left(\eta_{m}\right)-p_{0}\right)\right\|_{2+1 / 4, S^{T}} \\
& \leq \alpha_{3}\left(T^{1 / 2}\left\|u_{m}\right\|_{4, \Omega^{T}}, \sup _{t}\left\|\eta_{m}\right\|_{3, \Omega}\right) \\
& \quad \times\left(\int_{\Omega} \int_{0}^{T} \frac{\left|\int_{0}^{t}\left\|u_{m}\right\|_{4, \Omega} d \tau\right|^{2}+\left\|\eta_{m}\right\|_{3, \Omega}^{2}+\left\|u_{m}\right\|_{2, \Omega}^{2}+\left\|\eta_{m t}\right\|_{1, \Omega}^{2}}{t^{1 / 2}} d \xi d t\right)^{1 / 2} \\
& \quad \leq \alpha_{3} T^{1 / 4}\left(T^{1 / 2}\left\|u_{m}\right\|_{4, \Omega^{T}}+\sup _{t}\left\|\eta_{m}\right\|_{3, \Omega}+\sup _{t}\left\|\eta_{m t}\right\|_{1, \Omega}+\sup _{t}\left\|u_{m}\right\|_{2, \Omega}\right) .
\end{aligned}
$$

Finally, we consider the expression

$$
\bar{n}_{0} \cdot \Delta_{m}(t) \xi=\frac{1}{\sqrt{g}} \bar{n}_{0} \cdot g^{\alpha \beta} \partial_{s^{\alpha} s^{\beta}} \xi .
$$

Then we have

$$
\begin{aligned}
\left\|\bar{n}_{0} \cdot \Delta_{m}(t) \xi\right\|_{2+1 / 2, S^{T}} & \\
\leq & \alpha_{4}\left(T^{1 / 2}\left\|u_{m}\right\|_{4, \Omega^{T}}\right)\|\xi\|_{4+1 / 2, S}^{2} \\
& \times\left(T+\left\|u_{m}\right\|_{2, \Omega^{T}}^{2}+T^{1 / 2}\left\|u_{m}\right\|_{4, \Omega^{T}}^{2}+T\left\|u_{m}\right\|_{4, \Omega^{T}}^{4}\right),
\end{aligned}
$$

and

$$
\begin{aligned}
& \left\|\bar{n}_{0} \cdot \Delta_{m}(t) \xi\right\|_{2+1 / 2, S^{T}} \\
& \quad \leq \alpha_{5}\left(T^{1 / 2}\left\|u_{m}\right\|_{4, \Omega^{T}}\right)\|\xi\|_{4+1 / 2, S} T^{1 / 4}\left(T^{1 / 2}\left\|u_{m}\right\|_{4, \Omega^{T}}+\left\|u_{m}\right\|_{2, \Omega^{T}}\right) .
\end{aligned}
$$

Summarizing the above considerations and using Lemma 6.1 we have

$$
y_{m+1}(t) \leq \beta\left(t^{1 / 2} y_{m}(t), t, \gamma\right)+\alpha\left(t^{1 / 2} y_{m}(t), t, \gamma\right) \int_{0}^{t} y_{m+1}(\tau) d \tau
$$

where $\alpha, \beta$ are positive increasing functions and

$$
\begin{gathered}
y_{m}(t)=\left\|u_{m}\right\|_{4, \Omega^{t}}^{2}+\sup _{t}\left|u_{m}\right|_{3,0, \Omega}^{2}, \\
\gamma=\left\|\varrho_{0}\right\|_{3, \Omega}^{2}+\left|u_{m}(0)\right|_{3,0, \Omega}^{2} .
\end{gathered}
$$

In view of the Gronwall lemma we have

$$
\begin{aligned}
y_{m+1}(t) & \leq \exp \left[t \alpha\left(t^{1 / 2} y_{m}(t), t, \gamma\right)\right] \beta\left(t^{1 / 2} y_{m}(t), t, \gamma\right) \\
& \equiv G\left(\gamma, t^{1 / 2} y_{m}(t), t\right)
\end{aligned}
$$

where $G(\gamma, 0,0)=G_{0}(\gamma)>0$ and $G$ is an increasing positive function of its arguments.

Let $0<A$ be sufficiently large and such that $G_{0}(\gamma)<A, y_{m}(0)<A$. Then there exists a time $T_{*}$ such that for $t \leq T_{*}$ we have

$$
y_{m+1}(t) \leq G\left(\gamma, t^{1 / 2} A, t\right) \leq A
$$


In this way we have shown that

$$
y_{m}(t) \leq A \text { for } m=0,1, \ldots \text { and } t \leq T_{*} .
$$

Now we prove convergence of the sequence $\left\{u_{m}, \eta_{m}\right\}$. To show this we consider the system of problems for the differences $U_{m}=u_{m}-u_{m-1}$ and $H_{m}=\eta_{m}-\eta_{m-1}$ :

$$
\begin{aligned}
& \eta_{m} \partial_{t} U_{m+1}- \mu \nabla_{u_{m}}^{2} U_{m+1}-\nu \nabla_{u_{m}} \nabla_{u_{m}} \cdot U_{m+1} \\
&=-H_{m} \partial_{t} u_{m}-\mu\left(\nabla_{u_{m}}^{2}-\nabla_{u_{m-1}}^{2}\right) u_{m} \\
&-\nu\left(\nabla_{u_{m}} \nabla_{u_{m}} \cdot-\nabla_{u_{m-1}} \nabla_{u_{m-1}} \cdot\right) u_{m} \\
&+\nabla_{u_{m}} q\left(\eta_{m}\right)-\nabla_{u_{m-1}} q\left(\eta_{m-1}\right)+H_{m} g \equiv F_{1}+F_{2}, \\
& \Pi_{0} \mathbb{D}_{u_{m}}\left(U_{m+1}\right) \bar{n}\left(u_{m}\right) \\
&= \Pi_{0}\left[\mathbb{D}_{u_{m}}\left(u_{m}\right) \bar{n}\left(u_{m}\right)-\mathbb{D}_{u_{m-1}}\left(u_{m}\right) \bar{n}\left(u_{m-1}\right)\right] \equiv G_{1}, \\
& \bar{n}_{0} \mathbb{D}_{u_{m}}\left(U_{m+1}\right) \bar{n}\left(u_{m}\right)-\sigma \bar{n}_{0} \Delta_{m}(t) \int_{0}^{t} U_{m+1}(\tau) d \tau \\
&=\bar{n}_{0}\left[\mathbb{D}_{u_{m}}\left(u_{m}\right) \bar{n}\left(u_{m}\right)-\mathbb{D}_{u_{m-1}}\left(u_{m}\right) \bar{n}\left(u_{m-1}\right)\right] \\
& \quad-\sigma \bar{n}_{0}\left(\Delta_{m}(t)-\Delta_{m-1}(t)\right) \int_{0}^{t} u_{m}(\tau) d \tau \\
& \quad+\bar{n}_{0} \cdot\left[\bar{n}\left(u_{m}\right) q\left(\eta_{m}\right)-\bar{n}\left(u_{m-1}\right) q\left(\eta_{m-1}\right)\right] \\
& \quad-p_{0} \bar{n}_{0} \cdot\left(\bar{n}\left(u_{m}\right)-\bar{n}\left(u_{m-1}\right)\right) \\
& \quad+\sigma \bar{n}_{0}\left(\Delta_{m}(t)-\Delta_{m-1}(t)\right) \xi \equiv G_{2}+G_{3}, \\
& \quad
\end{aligned}
$$

where

$$
\begin{aligned}
F_{2} & =-H_{m} \partial_{t} u_{m}+H_{m} g+q^{\prime}\left(\widetilde{\eta}_{m}\right) \nabla_{u_{m}} H_{m}, \\
G_{3} & =\bar{n}\left(u_{m}\right)\left(q\left(\eta_{m}\right)-q\left(\eta_{m-1}\right)\right)
\end{aligned}
$$

and $F_{1}, G_{2}$ are determined by the remaining terms on the right-hand sides.

To estimate the right-hand sides of $(6.20)$ we shall restrict to their qualitative forms:

$$
\begin{aligned}
F_{1}= & f_{1} \int_{0}^{t} U_{m \xi} d \tau u_{m \xi \xi}+f_{2} \int_{0}^{t} U_{m \xi \xi} d \tau u_{m \xi} \\
& +f_{3} \int_{0}^{t} U_{m \xi} d \tau f_{1}^{\prime} \eta_{m-1, \xi} \\
G_{1}= & f_{4} \int_{0}^{t} U_{m \xi} d \tau u_{m \xi}, \\
G_{2}= & f_{5} \int_{0}^{t} U_{m \xi} d \tau\left(1+u_{m \xi}\right)+f_{2}^{\prime} \int_{0}^{t} U_{m \xi} d \tau,
\end{aligned}
$$


where $f_{i}=f_{i}\left(\delta+\int_{0}^{t} u_{m \xi} d \tau, \delta+\int_{0}^{t} u_{m-1 \xi} d \tau\right), i=1, \ldots, 6, f_{j}^{\prime}=f_{j}^{\prime}\left(\eta_{m}\right)$, $j=1,2$, are $C^{\infty}$ functions of their arguments. Moreover, we have

$$
\begin{aligned}
K & =-\bar{n}_{0}\left(\Delta_{m}(t)-\Delta_{m-1}(t)\right) u_{m} \\
& =f_{6}\left(\int_{0}^{t} U_{m \xi} d \tau u_{m \xi \xi}+\int_{0}^{t} U_{m \xi \xi} d \tau u_{m \xi}\right) .
\end{aligned}
$$

Now we have to estimate the functions (6.21)-(6.23):

$$
\begin{aligned}
& \left\|F_{2}\right\|_{2, \Omega^{T}} \leq C(A)\left(\sup _{t}\left\|H_{m}\right\|_{3, \Omega}+\sup _{t}\left\|H_{m t}\right\|_{1, \Omega}\right), \\
& \left\|F_{1}\right\|_{2, \Omega^{T}} \leq C(A) T^{1 / 2}\left(\left\|U_{m}\right\|_{4, \Omega^{T}}+\sup _{t}\left|U_{m}\right|_{3,0, \Omega}\right), \\
& \left\|G_{i}\right\|_{2+1 / 2, S^{T}} \leq C(A) T^{1 / 2}\left(\left\|U_{m}\right\|_{4, \Omega^{T}}+\sup _{t}\left|U_{m}\right|_{3,0, \Omega}\right) \quad i=1,2, \\
& \left\|G_{i}\right\|_{2+1 / 4, S^{T}} \leq C(A, T) T^{1 / 4}\left(\left\|U_{m}\right\|_{4, \Omega^{T}}+\sup _{t}\left|U_{m}\right|_{3,0, \Omega}\right), \\
& \|K\|_{2-1 / 2, S^{T}} \leq C(A) T^{1 / 2}\left(\left\|U_{m}\right\|_{4, \Omega^{T}}+\sup _{t}\left|U_{m}\right|_{3,0, \Omega}\right), \\
& \left.\left\|G_{3}\right\|_{2+1 / 2, S^{T}, 1 / 4} \leq C(A, T) T^{1 / 4} \sup _{t}\left\|H_{m}\right\|_{3, \Omega}+\sup _{t}\left\|H_{m t}\right\|_{1, \Omega}\right) .
\end{aligned}
$$

Summarizing the above considerations we have shown

$$
\begin{aligned}
\left\|U_{m+1}\right\|_{4, \Omega^{t}} \leq & \beta_{1}(T, A) T^{1 / 4}\left(\left\|U_{m}\right\|_{4, \Omega^{T}}+\sup _{t}\left|U_{m}\right|_{3,0, \Omega}\right) \\
& +\beta_{2}(T, A) \sup _{t}\left|H_{m}\right|_{3,0, \Omega} .
\end{aligned}
$$

Next, we consider the problem

$$
\begin{aligned}
& \partial_{t} H_{m}+H_{m} \operatorname{div}_{u_{m}} u_{m}=-\eta_{m-1}\left(\operatorname{div}_{u_{m}} u_{m}-\operatorname{div}_{u_{m-1}} u_{m-1}\right), \\
& \left.H_{m}\right|_{t=0}=0 .
\end{aligned}
$$

Integrating (6.26) with respect to time one obtains

$$
\begin{aligned}
& H_{m}(\xi, t) \\
& =-\exp \left[-\int_{0}^{t} \operatorname{div}_{u_{m}} u_{m} d \tau\right] \\
& \quad \times \int_{0}^{t}\left[\eta_{m-1}\left(\operatorname{div}_{u_{m}} u_{m}-\operatorname{div}_{u_{m-1}} u_{m-1}\right) \exp \int_{0}^{t^{\prime}} \operatorname{div}_{u_{m}} u_{m} d t^{\prime \prime}\right] d t^{\prime},
\end{aligned}
$$

so one has

$$
\sup _{t}\left|H_{m}\right|_{3,0, \Omega} \leq \beta_{3}(T, A) T^{1 / 2}\left\|U_{m}\right\|_{4, \Omega^{t}} .
$$

To obtain (6.28) the most difficult point is when we differentiate the middle term in (6.27) with respect to $t$. Then we have to estimate the ex- 
pression

$$
\begin{aligned}
\sup _{t} & \left\|\xi_{x}\left(u_{m-1}\right) U_{m \xi} \exp []\right\|_{1, \Omega} \\
& \leq \phi(A) \sup _{t}\left\|U_{m \xi}\right\|_{1, \Omega} \leq c \phi(A)\left\|U_{m \xi}\right\|_{2, \Omega^{t}} \\
& =c \phi(A)\left(\int_{0}^{t}\left(\left\|U_{m \xi}\right\|_{2, \Omega}^{2}+\left\|U_{m \xi t}\right\|_{0, \Omega}^{2}\right) d t\right)^{1 / 2} \\
& \leq c \phi(A) t^{1 / 2} \sup _{t}\left(\left\|U_{m \xi}\right\|_{2, \Omega}+\left\|U_{m t \xi}\right\|_{0, \Omega}\right) \leq c \phi(A) t^{1 / 2}\left\|U_{m}\right\|_{4, \Omega^{t}} .
\end{aligned}
$$

Now (6.25) and (6.28) imply that the sequence $\left\{u_{m}, \eta_{m}\right\}$ converges to a limit $\{u, \eta\} \in W_{2}^{4,2}\left(\Omega^{t}\right) \times L_{\infty}\left(0, t ; \Gamma_{0}^{3}(\Omega)\right)$ for $t \leq T_{* *}$, where $T_{* *}$ is sufficiently small. Uniqueness can be proved in the standard way. This concludes the proof.

\section{References}

[1] O. V. Besov, V. P. Il'in and S. M. Nikol'skiur, Integral Representation of Functions and Imbedding Theorems, Nauka, Moscow, 1975 (in Russian).

[2] D. K. Faddeev, V. A. Solonnikov and N. N. Ural'tseva, Selected Topics of Analysis and Algebra, Leningrad, 1981 (in Russian).

[3] O. A. Ladyzhenskaya, V. A. Solonnikov and N. N. Ural'tseva, Linear and Quasilinear Equations of Parabolic Type, Nauka, Moscow, 1967 (in Russian).

[4] L. Landau and E. Lifschitz, Mechanics of Continuum Media, Nauka, Moscow, 1954 (in Russian); English transl.: Pergamon Press, Oxford, 1959; new edition: Hydrodynamics, Nauka, Moscow, 1986 (in Russian).

[5] V. A. Solonnikov, On an initial-boundary value problem for the Stokes system which appears in free boundary problems, Trudy Mat. Inst. Steklov. 188 (1990), 150-188 (in Russian).

[6] - On boundary problems for linear parabolic systems of differential equations of general form, ibid. 83 (1965) (in Russian).

[7] —, A priori estimates for second order parabolic equations, ibid. 70 (1964), 133-212 (in Russian).

[8] V. A. Solonnikov and A. Tani, Free boundary problem for a viscous compressible flow with a surface tension, in: Constantine Carathéodory: An International Tribute, T. M. Rassias (ed.), World Scientific, 1991, 1270-1303.

[9] E. Zadrzyńska and W. M. Zajączkowski, On local motion of a general compressible viscous heat conducting fluid bounded by a free surface, Ann. Polon. Math. 59 (1994), 133-170.

[10] - - -, On the global existence theorem for a free boundary problem for equations of a viscous compressible heat conducting fluid, to appear.

[11] - - - On the global existence theorem for a free boundary problem for equations of a viscous compressible heat conducting capillary fluid, to appear.

[12] W. M. Zajączkowski, On an initial-boundary value problem for the parabolic system which appears in free boundary problems for compressible Navier-Stokes equations, Dissertationes Math. 304 (1990). 
[13] W. M. Zajączkowski, On local motion of a compressible barotropic viscous fluid bounded by a free surface, in: Banach Center Publ. 27, Inst. Math., Polish Acad. Sci., 1992, 511-553.

[14] -, On nonstationary motion of a compressible barotropic viscous fluid bounded by a free surface, Dissertationes Math. 324 (1993).

[15] - On nonstationary motion of a compressible barotropic viscous capillary fluid bounded by a free surface, SIAM J. Math. Anal. 25 (1994), 1-84.

INSTITUTE OF MATHEMATICS

POLISH ACADEMY OF SCIENCES

P.O. BOX 137

00-950 WARSZAWA, POLAND

Reçu par la Rédaction le 3.11.1993

Révisé le 15.3.1994 